\title{
ANTIMICROBIAL PHOTODYNAMIC THERAPY USING CURCUMIN PHOTOSENSITIZER IN CONJUNCTION WITH PERIODONTAL OPEN FLAP DEBRIDEMENT. PILOT CLINICAL STUDY WITH REAL-TIME PCR BACTERIAL ASSESSMENT
}

\author{
Ahmed Elsayed Hamed Amr*
}

\begin{abstract}
Aim: To evaluate the effect of using antimicrobial photodynamic therapy (A-PDT) with curcumin in conjunction with open flap debridement (OFD) in the treatment of deep periodontal pockets. The primary objective was the clinical evaluation via assessment of the changes in clinical periodontal parameters. The secondary objective was the quantification of the pocket total bacterial load by Real-Time PCR.

Subjects and methods: Group A included 7 patients who received OFD in conjunction with A-PDT using LED and curcumin paste (2\%) as a photosensitizer. Group B included 7 patients who received OFD followed by application of curcumin paste (2\%) but without photoactivation. Group C included 7 patients who received OFD alone. Clinical evaluation included plaque index (PI), gingival bleeding index (BI), probing depth (PD) and clinical attachment level (CAL). Microbiological evaluation involved collection of gingival crevicular fluid (GCF) samples for total bacterial load (TBL) analysis by real-time PCR
\end{abstract}

Results: Regarding the percent change in PD and CAL, the greatest percent decrease value was recorded in group A while the least percent decrease was in group C. The difference between groups was statistically significant. Regarding percent change of TBL by time, the greatest percent decrease value was recorded in group A.

Conclusion: Within the limitations of our study, the photoactivation of the curcumin used in conjunction with OFD lead to greater PD reduction, greater CAL gain and greater TBL reduction when compared to OFD alone or OFD with curcumin without photoactivation.

* Lecturer of Oral medicine, Periodontology and Oral diagnosis. Faculty of dentistry, Ain Shams University 


\section{INTRODUCTION}

Periodontal disease represents an infection where large number of microorganisms of different species share in its etiology (Rivera et al 2013). The periodontal pathogens are present in biofilms which are complex communities of microorganisms attached to the tooth surface (Kommerein et al 2017). The biofilm structure allows the pathogen to survive and resist antimicrobials (Koo et al 2017). Although the initiation of periodontal diseases is caused by microorganisms, the outcome is much affected by the immune response of the patient (Page et al 1997). The basic approach for management of periodontal diseases is the non-surgical mechanical removal of plaque and calculus by supragingival and subgingival scaling followed by root planing (Greenstein 2000, Heitz-Mayfield \& Lang 2013). Although significant improvement of many clinical parameters and biological markers of periodontal diseases have been achieved via scaling and root planing (Bender et al 2006, Flemmig \&Beikler 2011) but the complete eradication of the bacteria and bacterial toxins is so difficult to be reached (Adriaens \& Adriaens 2004). The presence of deep pockets, irregular complicated root anatomies and the presence of bacteria inside the tissues through bacterial invasion may compromise the efficiency of scaling and root planing (Heitz-Mayfield \& Lang 2013, How et al 2016). The use of complimentary antimicrobials systemically has been tried in many resistant cases. However, the use of systemic therapy is usually associated with potential side effects as allergy, gastrointestinal disturbances and bacterial resistance (Lopez et al 2000, Haffajee 2006, Woodford \& Ellington 2007). Moreover, in many cases the antibiotic concentration that reaches the gingival crevicular fluid (GCF) after systemic administration may be insufficient to inhibit the subgingival periopathogens (Darveau et al 1997).

Recently alternative therapeutic approaches have been introduced in the field of periodontics to control periodontal diseases with the aim of eradication of the periodontal pathogens. Among these approaches is the antimicrobial photodynamic therapy. The antimicrobial photodynamic therapy (A-PDT) is a safe, simple, effective and non-invasive modality in the management of periodontal diseases (Fekrazad et al 2017). The A-PDT is also known as photoactivated disinfection and photochemotherapy and was first presented by "Niels Finsen" a Danish physician in the management of skin tubercular condition called lupus vulgaris (Deniell \& Hill 1991). Contemporary A-PDT involves the application of a photosensitizer and its activation by a light source which have a specific wavelength that corresponds to the photosensitizer absorption maximum which in turn exerts antimicrobial effect (Abrahamse \& Hamblin 2016). The use of A-PDT in dentistry is growing rapidly and many applications have been tried. It has been applied in management of many cancerous lesions and different infections specially the resistant cases (Kornman et al 1997, Wainwright 1998, Sharwani et al 2006). The adjunctive use of A-PDT with non-surgical scaling and root planning for management of periodontitis has resulted in significant improvement in the clinical periodontal parameters (Andersen et al 2007, Alwaeli et al 2015, Malgikar et al 2016, Azaripour et al 2018). In addition to being non-invasive simple therapeutic approach, A-PDT is not associated with the bacterial resistance or the side effects associated with systemic antibiotics (Hamblin et al 2004). The action of A-PDT on microorganisms is mainly lethal rather than growth inhibition thus the appearance of resistance strains to A-PDT is rare (Konopka \& Goslinski 2007, Costa et al 2011, Parker 2013, Kashef \& Hamblin 2017). Also A-PDT can exert a detoxifying effect on bacterial LPs making the endotoxin unable to activate pro-inflammatory cytokines production by immune cells (Komerik et al 2000). The reaction associated with A-PDT occurs in limited space and for short time causing localized limited time response affecting the periopathogen without imposing any damage to the host cells owing to the protective presence of keratin (Rajesh 2011, 
Parker 2013). Moreover, A-PDT may accelerate the healing of periodontal tissue following scaling and root planning through stimulation of fibroblasts proliferation, collagen formation, new capillaries and bone formation (Konopka \& Goslinski 2007, Nesi-Reis et al 2018).

Basically, A-PDT depends on a light source and a photosensitizer in the presence of oxygen (MeyerBetz et al 1913).The light source is of utmost importance in A-PDT. Light between 630-700 nm has the power to activate photosensitizers (Grant et al 1997, Salva 2002). The helium-neon laser and argon laser are among the most common light sources in A-PDT (Juzeniene et al 2004). Sometimes light application induces heat generation at the site being treated but the application of liquid photosensitizers with lasers of low power level can manage this problem (Chen et al 2001, Chen et al 2002, Boehm \& Ciancio 2011). Being small size devices already present in each dental clinic and cheaper than laser devices, the light-emitting diodes (LED) have been applied in A-PDT as a non-laser light source for the photosensitizer activation (Takasaki et al 2009). The LED which is commonly present in dental clinics for curing composite resin have showed a phototoxic effect on periodontal pathogens at 400$500 \mathrm{~nm}$ (Feuerstein et al 2004). The broad band blue light sources (LED) at 380-520 nm can exert lethal action on black pigmented bacteria (Soukos et al 2005). Previous studies prove that LED in conjunction with a photosensitizer can significantly reduce bacterial load in periodontal pocket serving as a non-invasive antibacterial modality (O'Neill $\boldsymbol{e t}$ al 2002, Komerik \& MacRobert 2006).

Potential risks associated with A-PDT can be related to the photosensitizer used (Takasaki et al 2009, Rajesh et al 2011). Thus, in A-PDT it is highly important to use photosensitizers that allow killing the periopathogens without negatively affecting normal tissues (Bhatti et al 1998, Takasaki et al 2009, Rajesh et al 2011). The most popular photosensitizers used in the medical field are related to furocoumarins, tetrapyrroles and tricyclic dyes (Malik et al 2010). In A-PDT toluidine blue and methylene blue are the most used photosensitizers having similar physical, chemical criteria and having potent antibacterial actions (Lambrecht et al 1991, Millson et al 1996). Many of the photosensitizers have inherent antimicrobial activity but the bactericidal effects appear mainly after light activation (Kolenbrander 2000).

Turmeric (Curcuma Longa) was primarily known as a spice in food, coloring agent and among the home remedies (Ammon et al 1992). Many studies examined curcumin; the main bioactive yellow component of turmeric and showed many biological actions related to it. Curcumin has anti-inflammatory, antioxidant, anticarcinogenic, antidiabetic, antibacterial, antiviral and antifungal actions (Lampe \& Milobedzka 1913, Chattopadhyay et al 2004, kim et al 2005, Gulcubuk et al 2006, Jurenka 2009, Mun et al 2009, Moon et al 2010). Moreover, it stimulates new angiogenesis and collagen formation enhancing the healing of wounds (Komerik \& Wilson 2002). Studies documented that curcumin can be used as a potent photoactivatable material (Vera-Ramirez et al 2013, Wright et al 2013). It can be considered an ideal photosensitizer since its potential of singlet oxygen formation with excellent tissue biocompatibility and tolerability if ingested (Hatcher et al 2008, Araujo et al 2012). Significant antibacterial action of photoactivated curcumin has been reported in many previous studies (Araujo et al 2012, Paschoal et al 2013) through the formation of free radicals and reactive oxygen species promoting the phototoxicity (Chignell et al 1994, Khopde et al 2000). The antibacterial action of curcumin on the periodontal pathogens has been documented (Mahdi et al 2015).

Curcumin activity ranges from bacteriostatic to bactericidal for different pathogenic Gram-positive and Gram-negative bacteria (Negi et al., 1999; Magesh et al., 2013; Packiavathy et al., 2014). Curcumin inhibits Streptococcus mutans biofilm formation and suppress the bacterial attachment 
to teeth surfaces ( $\mathrm{Hu}$ et al., 2013a). Furthermore, bacterial membrane permeability with subsequent bacterial permanent damage can be induced by curcumin (Tyagi et al., 2015). Studies showed that curcumin inhibited the growth of many periodontal pathogens; Prevotella intermedia, Prophyromonas gingivalis, Fuso- bacterium nucleatum, and Treponema denticola (Bhatia et al., 2014; Izui et al., 2016). Moreover, Curcumin can be a potent host modulatory agent in periodontal disease management through its inhibitory effects on many cytokines which play an important role in the periodontal disease pathogenesis (Ranjan et al., 2004; Cho et al., 2007; Fahey et al., 2007; Cohen et al., 2009; Bakir et al., 2016). Additionally, curcumin inhibits lipopolysaccharide (LPS)-induced COX-2 expression, leading to a decreased synthesis of prostaglandin (PGE2), which is an important stimulator of bone resorption in periodontal diseases (Hong et al., 2004)

Periodontal open flap debridement (OFD) is a commonly used surgical periodontal treatment modality that allows enhanced visibility and accessibility for precise scaling and root planing in complicated cases resistant to conventional nonsurgical periodontal treatment (Kaldahl et al 1988, Canakci \& Canakci 2007). It allows better removal of plaque, bacterial endotoxins and diseased granulation tissue but without total eradication of the microorganisms in the diseased site (Schenk et al 2000, Carranza \& Takei 2010).

To the best of our knowledge, the use of curcumin/A-PDT in conjunction with OFD with clinical and microbiological assessment has not yet been evaluated in previous studies. Thus, taking in consideration the significant antibacterial, host modulatory effects and the photoactivatable potential of curcumin, our study was carried out to evaluate the use of photoactivated curcumin in conjunction with OFD aiming to introduce an alternative modality for treating deep periodontal pockets which may enhance the clinical and microbiological treatment outcomes.

\section{AIM OF THE STUDY}

The aim of the study was to evaluate the effect of using A-PDT with curcumin (photosensitizer) in conjunction with OFD in the treatment of deep periodontal pockets. The primary objective was the clinical evaluation via assessment of the changes in clinical periodontal parameters. The secondary objective was the quantification of the pocket total bacterial load by Real-Time PCR.

\section{SUBJECTS AND METHODS}

Twenty one patients were selected according to the study inclusion and exclusion criteria from the clinic of oral diagnosis, faculty of dentistry, Ain Shams University. The study was conducted in the form of PICO question (Patient "P", Intervention "I", Comparative "C", Outcome “O”). (Stone 2002) "P": Patient in the study should have periodontitis with at least single posterior true periodontal pocket of probing depth (PD) $\geq 5 \mathrm{~mm}$ and clinical attachment level (CAL) $\geq 3 \mathrm{~mm}$. "II": Application of A-PDT using LED and curcumin in conjunction with OFD. "C": OFD+Curcumin , OFD alone. "O": clinical \& microbiological outcomes. The study protocol was explained to the patients. All the patients signed an informed consent and were able perform proper home plaque control measures and showed good compliance with the study steps. The research ethics committee in the faculty of dentistry , Ain Shams University had reviewed and accepted the study treatment procedures ; approval number FDASU-Rec IM091605.

Inclusion criteria: Male or female, age range 35-45 years, systemically healthy according to burket's oral health history questionnaire (Glick et al 2008) and able to show again for follow-up. The patient should have at least single posterior periodontal pocket with $\mathrm{PD} \geq 5 \mathrm{~mm}$ and $\mathrm{CAL} \geq 3 \mathrm{~mm}$ evaluated 2 weeks after the conventional (phase one) periodontal therapy and associated with horizontal bone loss radiographically on bitewing films. On the day of surgery, beside the aforementioned PD 
and CAL, the patient should have plaque index $\leq 1$ (Silness \& Loe, 1964) and gingival bleeding index $\leq 1$ (Loe \& Silness 1967). Patients with large carious lesions or any periapical infections, smokers, pregnant females, patients with history of antibiotics or history of periodontal surgery the last six months were all excluded from the study (Haffajee et al 2003, Novak \& Novak 2010).

Patients grouping: Patients were allocated randomly by computer (Quick Calcs; GraphPad Software) into 3 groups. Group A included 7 patients who received open flap debridement (OFD) in conjunction with A-PDT using LED and curcumin paste $(2 \%)$ as a photosensitizer. Group B included 7 patients who received OFD followed by application of curcumin paste $(2 \%)$ but without photoactivation. Group $\mathbf{C}$ included 7 patients who received OFD alone.

Preparation of $2 \%$ curcumin gel by simple dispersion method: Carbopol-940 was soaked in purified water containing $0.2 \% \mathrm{w} / \mathrm{v}$ sodium benzoate overnight. Using tissue homogenizer hydroxypropyl methylcellulose (HPMC) solution was mixed in propylene glycol. $2 \mathrm{mg}$ of curcumin (Sigma Aldrich, St.louis, MO) was transferred into HPMC solution and homogenized. This drug solution was transferred to carbopol solution and homogenized. Triethanolamine was added quantity sufficient (q.s.) to neutralize the PH. Then, distilled water was added to make q.s. to $100 \mathrm{ml}$. The gel was stored at ambient temperature and protected from light. The formulation of $2 \%$ curcumin gel was prepared in faculty of pharmacy, Tanta University. This preparation showed increased bioavailability and a higher percentage of drug diffusion and good rheological and texture properties (Sharma et al 2012, Hosadurga et al 2014).

\footnotetext{
* NSK, ultrasonic scaler , Japan

** Hu-Friedy, universal curette, USA

*** Artinibsa, Inibsa, Spain

**** Woodpecker LED.D light cure unit, CHINA

****** D-tek sutures, Cyprus
}

Pre-operative preparation of the patients: Phase 1 periodontal treatment including nonsurgical supragingival, subgingival scaling and root planing was performed to all patients in the study 2 weeks before the day of surgery. Both ultrasonic scaling $^{*}$ and manual curettes* ${ }^{* *}$ had been used. Oral hygiene instructions were given to all patients.

Surgical therapy (Open flap debridement OFD): (Figure 1) After administration of local

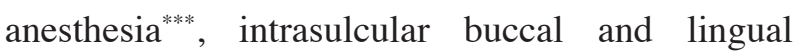
incisions were performed at the defect site extending one tooth mesial and one tooth distal to the defect in the three groups. This was followed by reflection of full thickness flap buccally and lingually exposing $3 \mathrm{~mm}$ of alveolar bone. Scalers, curettes and ultrasonic scalers were used for removal of plaque, calculus and any granulation tissues. After thorough debridement and saline irrigation, placement of cotton rolls for isolation was performed. Application of curcumin paste was then performed in group A followed by LED ${ }^{* * * *}$ activation for 5 minutes while in group B application of the curcumin paste alone was performed without light activation. In group C, OFD was performed alone without curcumin or light application. Interrupted sutures using $4 / 0$ polypropylene ${ }^{* * * * *}$ was then performed. Postoperative medications were then prescribed to all patients. Antibiotic : Amoxicillin/ Clavulanic acid orally $1 \mathrm{gm}$ every 12 hours for 7 days (Hibiotic, Amoun pharmaceutical, Egypt), Metronidazole $500 \mathrm{mg}$ orally tab every 12 hours for 7 days (Amrizole, pharco pharmaceutical, Egypt) ; Anti-inflammatory: diclofenac potassium $50 \mathrm{mg}$ tab every 8 hours for 3 days (Cataflam, Novartis, Egypt) and anti-edematous: chymotrypsin-trypsin, 1 tablet 3 times daily for 3 days (Alphintern,Amoun pharmaceutical, Egypt). All patients received 
postoperative instructions including rinsing with $0.12 \%$ chlorhexidine (Hexitol, $A D C O$, Egypt) twice daily for 2 weeks. Sutures were removed 2 weeks after the surgery. Phase 1 periodontal treatment, the surgical procedure, clinical measurements and bacterial sampling were performed by the author.

Clinical evaluation: Included plaque index $(\mathrm{PI})$, gingival bleeding index (BI), probing depth (PD) and clinical attachment level (CAL). Plaque Index (PI) (Silness \& Loe, 1964) as follows, 0: Gingival area of the tooth free of plaque, 1: No plaque is observed in situ by the unaided eye, but plaque is made visible on the tip of a probe after a probe has been moved over the tooth surface at the entrance of the gingival crevice, 2: Gingival area covered by a thin to moderately thick layer of plaque visible to the naked eye, 3: Heavy accumulation of soft matter, the thickness of which fills the gingival crevice. Gingival Bleeding Index (BI) (Loe \& Silness 1967) as follows, $0=$ Normal gingiva, 1 = Mild inflammation - slight change in color, slight edema, no bleeding on probing. $2=$ Moderate inflammation - redness, edema, glazing, bleeding on probing. $3=$ Severe inflammation marked redness and edema, ulceration, tendency toward spontaneous bleeding. The bleeding was assessed by probing gently along the wall of soft tissue of the gingival sulcus. Probing pocket depth (PD) (Caton, 1989); was measured from the gingival margin to the base of the periodontal pocket to the nearest $\mathrm{mm}$ using periodontal probe. Clinical attachment level (CAL) (Glavind \& Loe, 1967); was measured from the cemento-enamel junction (CEJ) to the base of the periodontal pocket to the nearest $\mathrm{mm}$ using periodontal probe. All measurements were taken using Williams graduated periodontal probe. All the above parameters were measured the day of the surgery just before starting the surgery (Baseline; PI1, BI1, PD1, CAL1) and 3 months following the periodontal surgery (Follow up; PI2, BI2, PD2, CAL2) (Azaripour et al 2018).

Microbiological evaluation: Involved collection of gingival crevicular fluid (GCF) samples for total bacterial load (TBL) analysis by real-time PCR (Theodoro et al 2012, Carneiro et al 2014). This was performed the day of the surgery just before starting the surgery (Baseline sample) and 1 month following the periodontal surgery (Follow up sample) (Magnusson et al 1984, Mombelli 2018). Before GCF collection, the site was isolated with cotton rolls and supragingival plaque was removed gently then the site was dried with gentle stream of air to avoid saliva contamination. Three endodontic paper points size 35 (Metabiomed, Korea ) were gently placed in the gingival sulcus till minor resistance was felt and left in place for 30 seconds (Wassall et al 2016) then the paper points was transferred to an eppendorf tube containing $1.5 \mathrm{ml}$ of phosphate buffered saline (7.4 $\mathrm{PH})$ as a reduced transport medium (Syed \& Loesche 1972). The transport medium preserves the sampled microorganisms. The sample was stored at $-80^{\circ} \mathrm{C}$ freezer till analysis.

Measurement of microbial DNA using quantitative Real time PCR (qPCR): The real time PCR was performed in "Global laboratories", Cairo, Egypt. Microbial DNA was extracted from GCF using Microbial DNA qPCR kit (Qiagen, Hilden, Germany). The bacterial DNA was extracted using DNA extraction and purification kit "QIAamp UCP Pathogen Mini Kit" (Qiagen, Hilden, Germany), then the concentration and purification was detected by determination of the A260/ A280 ratio. For amplification of microbial genes, a $25 \mu 1$ of reaction mix was prepared for each sample, it consists of 12.5 $\mu 1$ Microbial qPCR Mastermix, $1 \mu 1$ Microbial DNA qPCR assay sequence, $3 \mu 1$ of genomic DNA and $3.5 \mu 1$ of Microbial DNA-free water. The cycling protocol consists of (1): Initial PCR activation step at $95^{\circ} \mathrm{c}$ for 10 minutes, (2): two cycling step for 40 cycle, each cycle consists of $15 \mathrm{~s}$ at $95^{\circ} \mathrm{c}$ for DNA denaturation and $60^{\circ} \mathrm{c}$ for 2 minutes for primer annealing and extension. The amplification was performed using StepOne PCR instrument (Applied Biosystems, USA). For validation of the results, No Template Control (NTC), Microbial DNA Positive Control and Microbial DNA negative control was 
amplified parallel to the unknown sample. The calculation of bacterial load in each sample was determined using the Cycler specific software.

Statistical analysis was performed using a commercially available software program (SPSS 18; SPSS, Chicago, IL, USA). Data were explored for normality using Kolmogorov-Smirnov test of normality. Values of PI and BI were non-parametric and were expressed as median and range and compared between groups using Kruskall Wallis, while Wilcoxon-signed Rank test was used for intragroup comparisons. Values of PD, CAL, TBL and percent change of these parameters were normally distributed and were presented as mean, standard deviation (SD) and confidence intervals. ANOVA test was used for comparison between groups and was followed by Tukey's post hoc test if a significant difference was detected between groups. Paired (dependent) $\mathrm{t}$ test was used to compare baseline and follow-up values. The percentage of change was calculated by the following formula and the level of significance was set at $\mathrm{P} \leq 0.05$.

$$
\frac{\text { Value after-value before }}{\text { Value before }} \quad \text { X100 }
$$

\section{RESULTS}

\section{I-Plaque index (PI) and bleeding index (BI)}

\section{I-a-Comparison between groups}

Plaque index: At Baseline, Score 0 was recorded in all cases in the 3 groups, with no statistically significant difference $(\mathrm{p}=1)$. At follow-up, all groups showed a median value of 0 , ranging from 0 to 1 , with no significant difference between groups $(\mathrm{p}=0.815)$. Bleeding index: At Baseline, Group $\mathrm{A} \& \mathrm{~B}$ recorded a median value $=1$, ranging from 0 to 1 ; while group $\mathrm{C}$ recorded a median value $=0$, ranging from 0 to 1 , with no statistically significant difference between groups ( $\mathrm{p}=0.834)$. At follow-up, all groups showed a median value of 0 , ranging from 0 to 1 , with no statistically significant difference between groups $(\mathrm{p}=1)$. (table 1,2$)$

\section{I-b- Intragroup comparison}

Plaque index: In each group, the median value was $=0$ at baseline and at follow up, with no significant difference between baseline and followup. Bleeding index: In group $\mathrm{A} \& \mathrm{~B}$, the median value was $=1$ at baseline and decreased to 0 at follow up, with a range between 0 and 1 in both observation
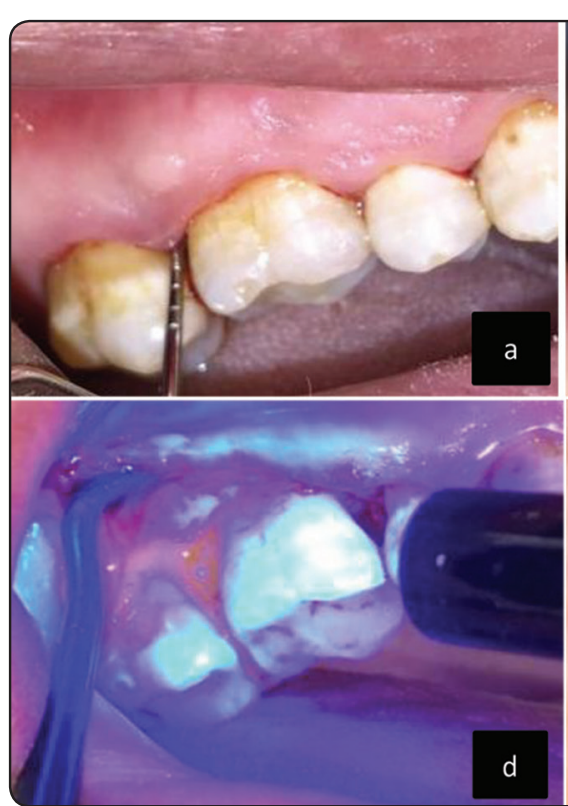
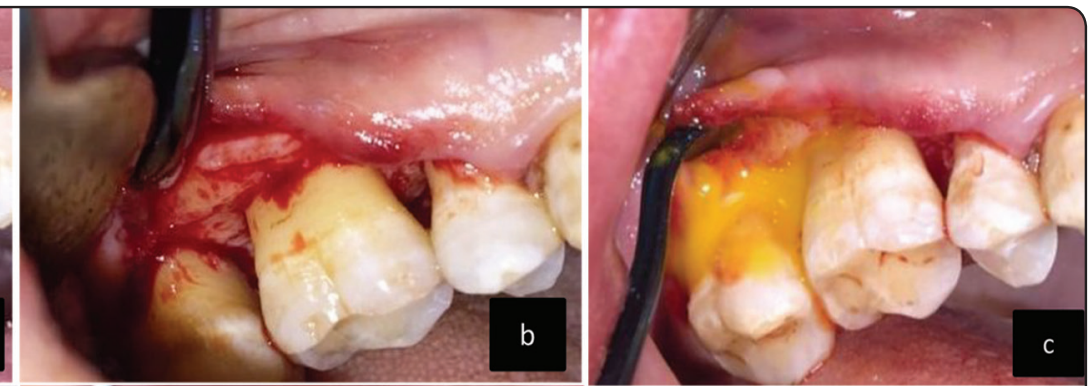

Fig. (1)

a. PD/CAL evaluation

b. Horizontal defect

c. Curcumin Application

d. LED photoactivation

e. Interrupted sutures 
times, with no significant difference between baseline and follow-up. Group $\mathrm{C}$ recorded at median of $=0$, ranging from 0 to 1 in both observation times, with no significant difference between baseline and follow-up. (table 1,2)

TABLE (1) Descriptive statistics [median (min, max.)] and comparison of PI and BI (Wilcoxon-signed Rank test for intragroup and Kruskall Wallis test for intergroup comparison)

\begin{tabular}{|c|c|c|c|c|}
\hline & & Baseline & Follow up & P (intragroup) \\
\hline \multirow{4}{*}{ PI } & Group A & $0(0,0)$ & $0(0,1)$ & $.157 \mathrm{~ns}$ \\
\cline { 2 - 5 } & Group B & $0(0,0)$ & $0(0,1)$ & $.083 \mathrm{~ns}$ \\
\cline { 2 - 5 } & Group C & $0(0,0)$ & $0(0,1)$ & $.157 \mathrm{~ns}$ \\
\cline { 2 - 5 } & P (intergroup) & $1 \mathrm{~ns}$ & $0.815 \mathrm{~ns}$ & \\
\hline \multirow{4}{*}{ BI } & Group A & $1(0,1)$ & $0(0,1)$ & $.157 \mathrm{~ns}$ \\
\cline { 2 - 5 } & Group B & $1(0,1)$ & $0(0,1)$ & $.157 \mathrm{~ns}$ \\
\cline { 2 - 5 } & Group C & $0(0,1)$ & $0(0,1)$ & $.317 \mathrm{~ns}$ \\
\cline { 2 - 5 } & P (intergroup) & $0.834 \mathrm{~ns}$ & $1 \mathrm{~ns}$ & \multicolumn{3}{|l}{} \\
\hline \multicolumn{4}{|c}{} & \\
\hline
\end{tabular}

Significance level $p \leq 0.05, n s=n o n-$ significant

\section{II- Probing depth (PD) and Clinical Attachment level (CAL)}

\section{II-a-Comparison between groups}

Probing depth: At baseline, there was no significant difference between groups $(\mathrm{p}=0.91)$. At follow up, the highest mean value was recorded in group $\mathrm{C}$, followed by group $\mathrm{B}$, with the least mean value recorded in group A. ANOVA test revealed a significant difference between groups $(p=0.00)$. Tukey's post hoc test showed that the difference between each 2 groups was statistically significant. Regarding PD at follow up, the mean difference between groups A\&B was [-2 (confidence Interval -3.29 to -0.71$)$ ], with a statistically significant difference $(\mathrm{p}=0.003)$. The mean difference between groups $\mathrm{A} \& \mathrm{C}$ was $[-3.86$ (confidence Interval -5.15 to -2.57)], with a statistically significant difference $(\mathrm{p}=0.00)$. The mean difference between groups $\mathrm{B} \& \mathrm{C}$ was -1.86 (confidence Interval -3.15 to -0.56 )], with a statistically significant difference $(\mathrm{p}=0.005)$. (Fig.2, Table 3,4)

TABLE (2) Mean difference and confidence intervals of difference between groups regarding the PI and BI

\begin{tabular}{|c|c|c|c|c|c|c|}
\hline \multirow{2}{*}{ Variable } & \multirow{2}{*}{ (I) Groups } & \multirow{2}{*}{ (J) Groups } & \multirow{2}{*}{$\begin{array}{c}\text { Mean Difference } \\
(\mathrm{I}-\mathrm{J})\end{array}$} & \multicolumn{2}{|c|}{$95 \%$ Confidence Interval } & \multirow{2}{*}{$\mathrm{P}$} \\
\hline & & & & Lower Bound & Upper Bound & \\
\hline \multirow{3}{*}{$\begin{array}{c}\text { PI } \\
\text { baseline }\end{array}$} & \multirow{2}{*}{ Group A } & Group B & 0 & 0 & 0 & $1.000 \mathrm{~ns}$ \\
\hline & & Group C & 0 & 0 & 0 & $1.000 \mathrm{~ns}$ \\
\hline & Group B & Group C & 0 & 0 & 0 & $1.000 \mathrm{~ns}$ \\
\hline \multirow{3}{*}{$\begin{array}{c}\text { PI. } \\
\text { follow_up }\end{array}$} & \multirow{2}{*}{ Group A } & Group B & -.14286 & -.8303 & .5446 & $.858 \mathrm{~ns}$ \\
\hline & & Group C & .00000 & -.6875 & .6875 & $1.000 \mathrm{~ns}$ \\
\hline & Group B & Group C & .14286 & -.5446 & .8303 & $.858 \mathrm{~ns}$ \\
\hline \multirow{3}{*}{$\begin{array}{c}\text { BI } \\
\text { baseline }\end{array}$} & \multirow{2}{*}{ Group A } & Group B & .00000 & -.7292 & .7292 & $1.000 \mathrm{~ns}$ \\
\hline & & Group C & .14286 & -.5863 & .8720 & $.872 \mathrm{~ns}$ \\
\hline & Group B & Group C & .14286 & -.5863 & .8720 & $.872 \mathrm{~ns}$ \\
\hline \multirow{3}{*}{$\begin{array}{c}\text { BI } \\
\text { follow_up }\end{array}$} & \multirow{2}{*}{ Group A } & Group B & .00000 & -.6657 & .6657 & $1.000 \mathrm{~ns}$ \\
\hline & & Group C & .00000 & -.6657 & .6657 & $1.000 \mathrm{~ns}$ \\
\hline & Group B & Group C & .00000 & -.6657 & .6657 & $1.000 \mathrm{~ns}$ \\
\hline
\end{tabular}


Clinical Attachment level: At baseline, there was no significant difference between groups $(\mathrm{p}=0.47)$. At follow up, the highest mean value was recorded in group $\mathrm{C}$, followed by group $\mathrm{B}$, with the least mean value recorded in group $\mathrm{A}$. ANOVA test revealed a significant difference between groups $(\mathrm{p}=0.00)$. Tukey's post hoc test showed that the difference between each 2 groups was statistically significant. Regarding CAL at follow up, The mean difference between groups A\&B was [-1.71 (confidence Interval -2.8 to -0.62 )], with a statistically significant difference $(\mathrm{p}=0.002)$. The mean difference between groups $\mathrm{A} \& \mathrm{C}$ was
[-3.29 (confidence Interval -4.37 to -2.2$)]$, with a statistically significant difference $(\mathrm{p}=0.00)$. The mean difference between groups $\mathrm{B} \& \mathrm{C}$ was -1.57 (confidence Interval -2.66 to -0.48 )], with a statistically significant difference $(\mathrm{p}=0.005)$. (Fig.3, Table 3,4 )

\section{II-b- Intragroup Comparison}

For groups $\mathrm{A}$ and $\mathrm{B}$ : The mean value significantly decreased after treatment $(\mathrm{P}=0.00$ for $\mathrm{PD}$ and $\mathrm{CAL})$, group $\mathrm{C}$ : The mean value significantly decreased after treatment $(\mathrm{P}=0.00$ for $\mathrm{PD}$ and $\mathrm{p}=0.03$ for $\mathrm{CAL})$ (Table 5, Fig.2-3).

TABLE (3) Descriptive statistics and comparison of PD and CAL between groups (ANOVA test)

\begin{tabular}{|c|c|c|c|c|c|c|c|c|c|c|}
\hline & & & & & $\begin{array}{l}95 \% \\
\text { Interva }\end{array}$ & $\begin{array}{l}\text { idence } \\
\text { r Mean }\end{array}$ & & & & \\
\hline & & UP & Mean & Sta. Deviation & $\begin{array}{l}\text { Lower } \\
\text { Bound }\end{array}$ & $\begin{array}{l}\text { Upper } \\
\text { Bound }\end{array}$ & Minn & $\operatorname{Max}$ & $\mathrm{F}$ & $P$ \\
\hline & & A & 7.29 & 1.11 & 6.26 & 8.31 & 6.00 & 9.00 & & \\
\hline & Baseline & B & 7.00 & 1.63 & 5.49 & 8.51 & 5.00 & 9.00 & .10 & $.91 \mathrm{~ns}$ \\
\hline י & & $\mathrm{C}$ & 7.29 & 1.38 & 6.01 & 8.56 & 5.00 & 9.00 & & \\
\hline & & A & $1.86^{\mathrm{c}}$ & .38 & 1.51 & 2.21 & 1.00 & 2.00 & & \\
\hline & Follow_up & B & $3.86^{\mathrm{b}}$ & .99 & 2.94 & 4.77 & 2.50 & 5.00 & 29.04 & $.00 *$ \\
\hline & & $\mathrm{C}$ & $5.71^{\mathrm{a}}$ & 1.25 & 4.55 & 6.87 & 4.00 & 7.00 & & \\
\hline & & A & 4.29 & .95 & 3.41 & 5.17 & 3.00 & 5.00 & & \\
\hline CAL & Baseline & B & 4.71 & 1.11 & 3.69 & 5.74 & 3.00 & 6.00 & .78 & $.47 \mathrm{~ns}$ \\
\hline & & $\mathrm{C}$ & 5.00 & 1.15 & 3.93 & 6.07 & 3.00 & 6.00 & & \\
\hline & & A & $1.14^{\mathrm{c}}$ & .69 & .50 & 1.78 & .00 & 2.00 & & \\
\hline & Follow_up & B & $2.86^{\mathrm{b}}$ & .90 & 2.03 & 3.69 & 1.50 & 4.00 & 29.78 & $.00 *$ \\
\hline & & $\mathrm{C}$ & $4.43^{\mathrm{a}}$ & .79 & 3.70 & 5.16 & 3.00 & 5.00 & & \\
\hline
\end{tabular}

Significance level $p \leq 0.05$, *significant, $n s=$ non-significant, 
TABLE (4) Mean difference and confidence intervals of difference between groups regarding the PD and CAL

\begin{tabular}{|c|c|c|c|c|c|c|}
\hline \multirow{2}{*}{$\begin{array}{l}\text { Dependent } \\
\text { Variable }\end{array}$} & \multirow{2}{*}{ (I) Groups } & \multirow{2}{*}{ (J) Groups } & \multirow{2}{*}{$\begin{array}{c}\text { Mean Difference } \\
\text { (I-J) }\end{array}$} & \multicolumn{2}{|c|}{ 95\% Confidence Interval } & \multirow[b]{2}{*}{$\mathrm{P}$} \\
\hline & & & & $\begin{array}{l}\text { Lower } \\
\text { Bound }\end{array}$ & Upper Bound & \\
\hline \multirow{3}{*}{$\begin{array}{c}\text { PD } \\
\text { baseline }\end{array}$} & \multirow{2}{*}{ Group A } & Group B & .28571 & -1.6127 & 2.1841 & $.922 \mathrm{~ns}$ \\
\hline & & Group C & .00000 & -1.8984 & 1.8984 & $1.000 \mathrm{~ns}$ \\
\hline & Group B & Group C & -.28571 & -2.1841 & 1.6127 & $.922 \mathrm{~ns}$ \\
\hline \multirow{3}{*}{$\begin{array}{c}\text { PD } \\
\text { follow_up }\end{array}$} & \multirow{2}{*}{ Group A } & Group B & $-2.00000^{*}$ & -3.2919 & -.7081 & $.003 *$ \\
\hline & & Group C & $-3.85714 *$ & -5.1490 & -2.5652 & $.000 *$ \\
\hline & Group B & Group C & $-1.85714^{*}$ & -3.1490 & -.5652 & $.005^{*}$ \\
\hline \multirow{3}{*}{$\begin{array}{c}\text { AL } \\
\text { Baseline }\end{array}$} & \multirow{2}{*}{ Group A } & Group B & -.42857 & -1.8970 & 1.0399 & $.741 \mathrm{~ns}$ \\
\hline & & Group C & -.71429 & -2.1828 & .7542 & $.445 \mathrm{~ns}$ \\
\hline & Group B & Group C & -.28571 & -1.7542 & 1.1828 & $.874 \mathrm{~ns}$ \\
\hline \multirow{3}{*}{$\begin{array}{c}\text { AL } \\
\text { follow_up }\end{array}$} & \multirow{2}{*}{ Group A } & Group B & $-1.71429 *$ & -2.8013 & -.6273 & $.002 *$ \\
\hline & & Group C & $-3.28571 *$ & -4.3727 & -2.1987 & $.000^{*}$ \\
\hline & Group B & Group C & $-1.57143 *$ & -2.6584 & -.4844 & $.005^{*}$ \\
\hline
\end{tabular}

Significance level $p \leq 0.05, \quad \quad *$ significant, $\quad n s=n o n$-significant

TABLE (5) Comparison between baseline and follow up values of PD and CAL within each group

\begin{tabular}{|c|c|c|c|c|c|c|c|c|c|}
\hline & & Value & & & an change & om base & & & \\
\hline & & & & & & & & $\mathrm{t}$ & $\mathrm{P}$ \\
\hline & & & & & & Lower & Upper & & \\
\hline & PD & $7.29 \pm 1.11$ & $1.86 \pm 0.38$ & 5.43 & 1.13 & 4.38 & 6.48 & 12.67 & $.00 *$ \\
\hline & CAL & $4.29 \pm 0.95$ & $1.14 \pm 0.69$ & 3.14 & .90 & 2.31 & 3.97 & 9.24 & $.00 *$ \\
\hline & PD & $7.00 \pm 1.63$ & $3.86 \pm 0.99$ & 3.14 & .80 & 2.40 & 3.88 & 10.37 & $.00 *$ \\
\hline & CAL & $4.71 \pm 1.11$ & $2.86 \pm 0.9$ & 1.86 & .69 & 1.22 & 2.50 & 7.12 & $.00 *$ \\
\hline & PD & $7.29 \pm 1.38$ & $5.71 \pm 1.25$ & 1.57 & .35 & 1.25 & 1.89 & 12.05 & $.00 *$ \\
\hline & CAL & $5.00 \pm 1.15$ & $4.43 \pm 0.79$ & .57 & .53 & .08 & 1.07 & 2.83 & $.03 *$ \\
\hline
\end{tabular}




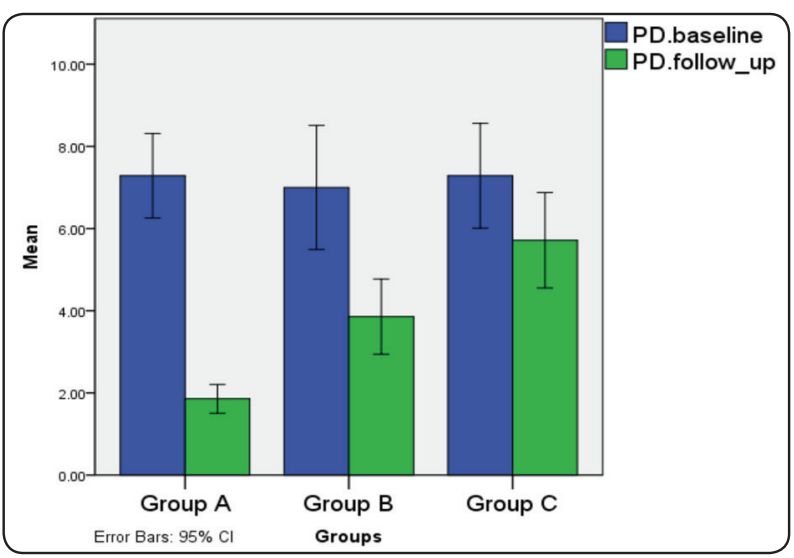

Fig. (2) Bar chart of mean PD at baseline and follow-up

\section{II -c-Comparison of percent change in PD and CAL by time}

Probing depth: The greatest percent decrease value was recorded in group A, while the least percent decrease was in group C. ANOVA test revealed that the difference between groups was statistically significant $(\mathrm{p}=0.000)$. Post hoc test revealed a significant difference between each 2 groups. Regarding percent change from baseline for $\mathrm{PD}$, The mean difference between groups A\&B was [-29.00 (confidence Interval -31.17 to -20.82$)$ ], with a statistically significant difference $(\mathrm{p}=0.00)$. The mean difference between groups A\&C was [-52.15 (confidence Interval -60.32 to -43.97$)$ ], with a statistically significant difference $(\mathrm{p}=0.00)$. The mean difference between groups $\mathrm{B} \& \mathrm{C}$ was -23.15 (confidence Interval -31.33 to -14.97$)$ ], with a statistically significant difference $(\mathrm{p}=0.00)$. (Table 6,7)

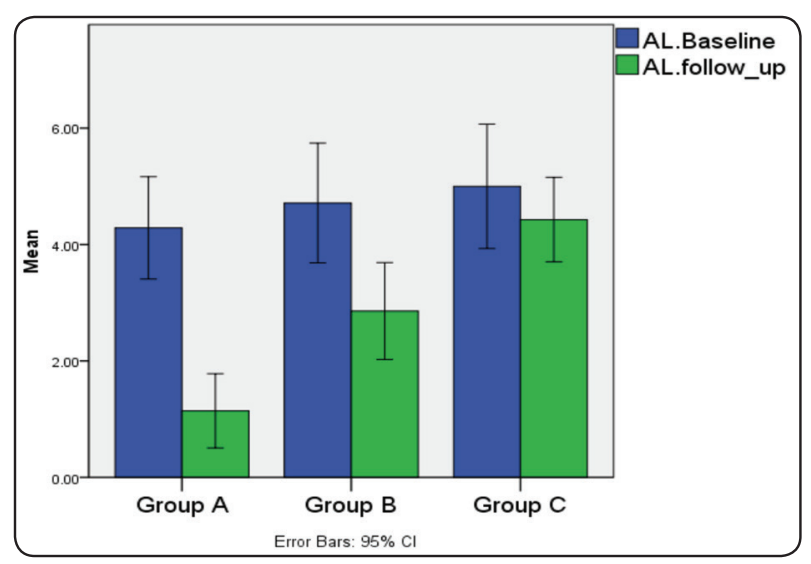

Fig. (3) Bar chart of mean CAL at baseline and follow-up

Clinical Attachment level: The greatest percent decrease value was recorded in group A, while the least percent decrease was in group C. ANOVA test revealed that the difference between groups was statistically significant $(\mathrm{p}=0.000)$. Post hoc test revealed a significant difference between each 2 groups. Regarding percent change from baseline for CAL, The mean difference between groups A\&B was [-33.93 (confidence Interval -51.4 to -16.46)], with a statistically significant difference $(\mathrm{p}=0.00)$. The mean difference between groups $\mathrm{A} \& \mathrm{C}$ was [-63.81 (confidence Interval -81.28 to -46.34)], with a statistically significant difference $(\mathrm{p}=0.00)$. The mean difference between groups $\mathrm{B} \& \mathrm{C}$ was -29.88 (confidence Interval -47.35 to -12.41 )], with a statistically significant difference $(\mathrm{p}=0.001)$. (Table 6,7)

TABLE (6) Comparison of percent change of PD and CAL between groups (ANOVA test)

\begin{tabular}{|c|c|c|c|c|c|c|c|c|c|}
\hline & & \multirow[t]{2}{*}{ Mean } & \multirow[t]{2}{*}{ Std. Dev } & \multicolumn{2}{|c|}{$\begin{array}{l}95 \% \text { Confidence Interval } \\
\text { for Mean } \\
\end{array}$} & \multirow[t]{2}{*}{ Min } & \multirow[t]{2}{*}{ Max. } & \multirow[t]{3}{*}{$\mathrm{F}$} & \multirow[t]{3}{*}{$\mathrm{P}$} \\
\hline \multicolumn{2}{|c|}{ Group } & & & Lower & Upper & & & & \\
\hline \multirow{3}{*}{ PD } & A & $-74.04^{\mathrm{a}}$ & 6.68 & -80.21 & -67.86 & -85.71 & -66.67 & & \\
\hline & $\mathrm{B}$ & $-45.04^{\mathrm{b}}$ & 5.98 & -50.57 & -39.51 & -50.00 & -33.33 & 133.02 & $0.00^{*}$ \\
\hline & $\mathrm{C}$ & $-21.89^{\mathrm{c}}$ & 5.23 & -26.73 & -17.05 & -33.33 & -18.75 & & \\
\hline \multirow{3}{*}{ CAL } & A & $-73.81^{\mathrm{a}}$ & 16.38 & -88.96 & -58.66 & -100.00 & -50.00 & 43.51 & $0.00 *$ \\
\hline & $\mathrm{B}$ & $-39.88^{\mathrm{b}}$ & 11.61 & -50.62 & -29.14 & -50.00 & -20.00 & & \\
\hline & C & $0.00^{\mathrm{c}}$ & 9.43 & -18.72 & -1.28 & -20.00 & .00 & & \\
\hline
\end{tabular}


TABLE (7) Mean difference and confidence intervals of difference between groups regarding the percent change from baseline of PD and CAL

\begin{tabular}{|c|c|c|c|c|c|c|}
\hline \multirow{2}{*}{$\begin{array}{c}\text { Dependent } \\
\text { Variable }\end{array}$} & \multirow{2}{*}{ (I) Groups } & \multirow[b]{2}{*}{ (J) Groups } & \multirow{2}{*}{$\begin{array}{c}\text { Mean } \\
\text { Difference } \\
(\mathrm{I}-\mathrm{J})\end{array}$} & \multicolumn{2}{|c|}{ 95\% Confidence Interval } & \multirow[b]{2}{*}{ Sig. } \\
\hline & & & & $\begin{array}{l}\text { Lower } \\
\text { Bound }\end{array}$ & Upper Bound & \\
\hline \multirow{3}{*}{$\begin{array}{c}\text { PD } \\
\text { Percent } \\
\text { change }\end{array}$} & \multirow{2}{*}{ Group A } & Group B & $-28.99660 *$ & -37.1730 & -20.8202 & $.000 *$ \\
\hline & & Group C & $-52.14569 *$ & -60.3221 & -43.9693 & $.000 *$ \\
\hline & Group B & Group C & $-23.14909 *$ & -31.3255 & -14.9727 & $.000 *$ \\
\hline \multirow{3}{*}{$\begin{array}{c}\text { AL } \\
\text { percent } \\
\text { change }\end{array}$} & \multirow{2}{*}{ Group A } & Group B & $-33.92857 *$ & -51.3981 & -16.4591 & $.000 *$ \\
\hline & & Group C & $-63.80952 *$ & -81.2790 & -46.3400 & $.000 *$ \\
\hline & Group B & Group C & $-29.88095^{*}$ & -47.3505 & -12.4114 & $.001 *$ \\
\hline
\end{tabular}

Significance level $p \leq 0.05$, *significant

\section{III- Total bacterial load (TBL)}

\section{III-a-Comparison between groups}

At baseline, there was no significant difference between groups ( $\mathrm{p}=0.91)$. At follow up, the highest mean value was recorded in group $\mathrm{C}$, followed by group $\mathrm{B}$, with the least mean value recorded in group A. ANOVA test revealed a significant difference between groups $(\mathrm{p}=0.00)$. Tukey's post hoc test showed that the difference between each 2 groups was statistically significant Regarding TBL at follow up, the mean difference between groups A\&B was [-1303.57(confidence Interval -1827.26 to -734.88)], with a statistically significant difference $(\mathrm{p}=0.00)$. The mean difference between groups A\&C was [-2782.14 (confidence Interval -3350.83 to -2213.45)], with a statistically significant difference $(p=0.00)$. The mean difference between groups $\mathrm{B} \& \mathrm{C}$ was -1478.57 (confidence Interval -2047.26 to -909.88 )], with a statistically significant difference $(\mathrm{p}=0.00)$. Regarding percent change of
TBL by time, the greatest percent decrease value was recorded in group A, while the least percent decrease was in group C. ANOVA test revealed that the difference between groups was statistically significant $(\mathrm{p}=0.000)$. Post hoc test revealed a significant difference between each 2 groups . Regarding the percent change of TBL from baseline, the mean difference between groups $A \& B$ was [-24.81(confidence Interval -30.07 to -19.55 )], with a statistically significant difference $(\mathrm{p}=0.00)$. The mean difference between groups $\mathrm{A} \& \mathrm{C}$ was [-52.72 (confidence Interval -57.98 to -47.46 )], with a statistically significant difference $(\mathrm{p}=0.00)$. The mean difference between groups $\mathrm{B} \& \mathrm{C}$ was -27.91 (confidence Interval -33.17 to -22.64 )], with a statistically significant difference $(\mathrm{p}=0.00)$. (Table 8,9)

\section{III-b- Intragroup Comparison}

For the three groups, the mean value significantly decreased after treatment $(\mathrm{P}=0.00)$. (Table 10) 
TABLE (8) Comparison of values and percent change of TBL between groups (ANOVA test)

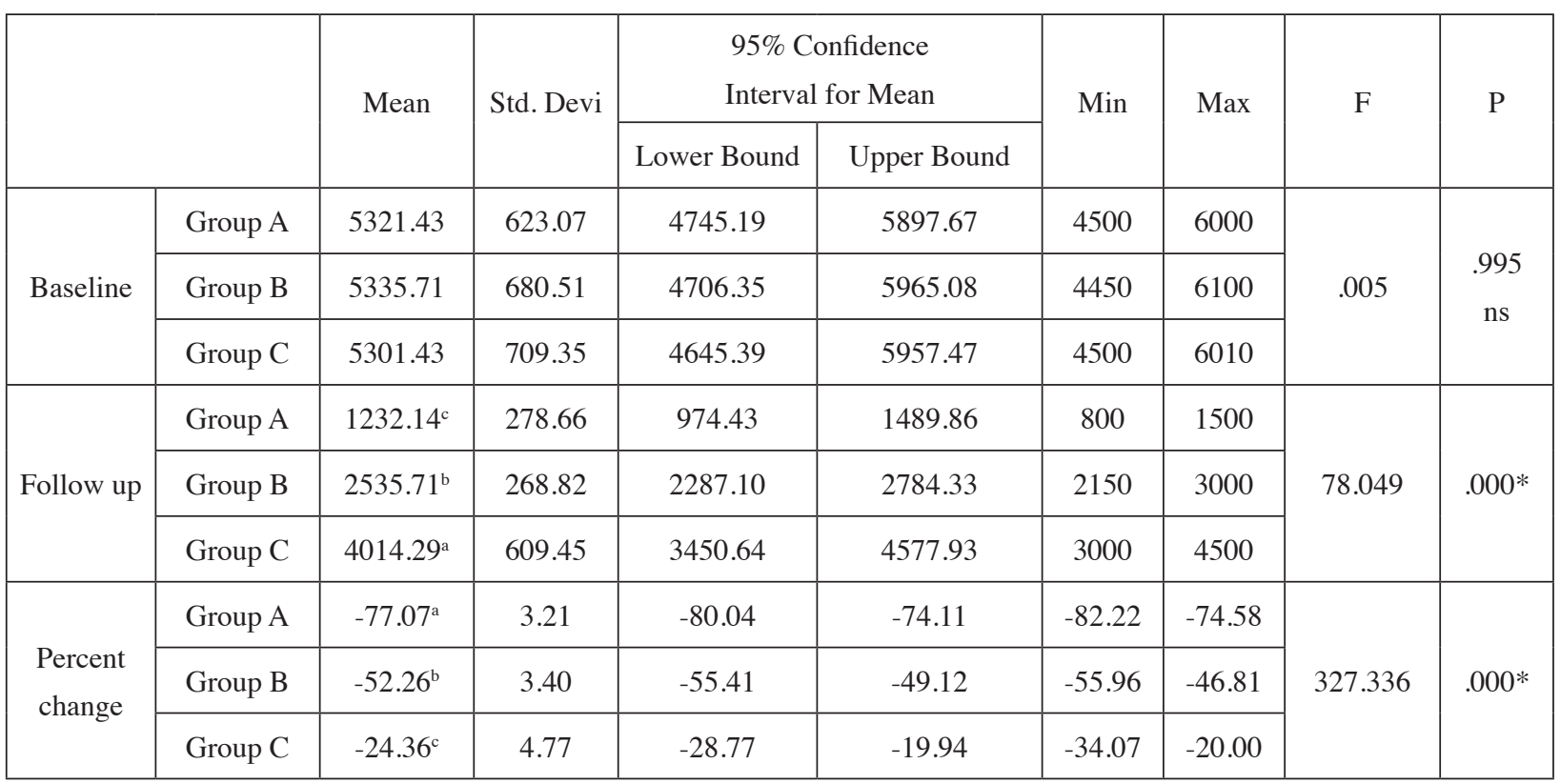

Significance level $p \leq 0.05$, *significant, ns=non-significant, Tukey's post hoc test: Within the same comparison, means sharing the same superscript letter are not significantly different

TABLE (9) Mean difference and confidence intervals of difference between groups regarding TBL and the percent change from baseline of TBL

\begin{tabular}{|c|c|c|c|c|c|c|}
\hline \multirow{2}{*}{ Variable } & \multirow{2}{*}{ (I) Groups } & \multirow{2}{*}{ (J) Groups } & \multirow{2}{*}{$\begin{array}{c}\text { Mean Difference } \\
\text { (I-J) }\end{array}$} & \multicolumn{2}{|c|}{ 95\% Confidence Interval } & \multirow{2}{*}{ Sig. } \\
\hline & & & & Lower Bound & Upper Bound & \\
\hline \multirow{3}{*}{$\begin{array}{c}\text { TBL } \\
\text { baseline }\end{array}$} & \multirow{2}{*}{ Group A } & Group B & -14.28571 & -930.9320 & 902.3605 & $.999 \mathrm{~ns}$ \\
\hline & & Group C & 20.00000 & -896.6463 & 936.6463 & $.998 \mathrm{~ns}$ \\
\hline & Group B & Group C & 34.28571 & -882.3605 & 950.9320 & $.995 \mathrm{~ns}$ \\
\hline \multirow{3}{*}{$\begin{array}{c}\text { TBL } \\
\text { Follow-up }\end{array}$} & \multirow{2}{*}{ Group A } & Group B & $-1303.57143 *$ & -1872.26 & -734.8835 & $.000 *$ \\
\hline & & Group C & $-2782.14286^{*}$ & -3350.83 & -2213.4549 & $.000 *$ \\
\hline & Group B & Group C & $-1478.57143 *$ & -2047.26 & -909.8835 & $.000 *$ \\
\hline \multirow{3}{*}{$\begin{array}{c}\text { Percent } \\
\text { TBL }\end{array}$} & \multirow{2}{*}{ Group A } & Group B & $-24.812^{*}$ & -30.07 & -19.55 & $.000 *$ \\
\hline & & Group C & $-52.717^{*}$ & -57.98 & -47.46 & $.000 *$ \\
\hline & Group B & Group C & $-27.905^{*}$ & -33.17 & -22.64 & $.000 *$ \\
\hline
\end{tabular}

Significance level $p \leq 0.05$, *significant 
TABLE (10) Comparison between baseline and follow up of TBL within each group (paired t test)

\begin{tabular}{|c|c|c|c|c|c|c|c|c|}
\hline \multirow{3}{*}{ Groups } & \multicolumn{2}{|c|}{ Value } & \multicolumn{4}{|c|}{ Mean change from baseline } & \multirow{3}{*}{$\mathrm{t}$} & \multirow{3}{*}{$\mathrm{P}$} \\
\hline & \multirow{2}{*}{ Baseline } & \multirow{2}{*}{ Follow-up } & \multirow{2}{*}{ Mean } & \multirow{2}{*}{ Std. Dev } & \multicolumn{2}{|c|}{ 95\% C.I. } & & \\
\hline & & & & & Lower & Upper & & \\
\hline Group A & $5321.43 \pm 623.07$ & $1232.14 \pm 278.66$ & 4089.29 & 381.84 & 3736.14 & 4442.43 & 28.33 & $.00 *$ \\
\hline Group B & $5335.71 \pm 680.51$ & $2535.71 \pm 268.82$ & 2800.00 & 478.71 & 2357.26 & 3242.74 & 15.48 & $.00 *$ \\
\hline Group C & $5301.43 \pm 709.35$ & $4014.29 \pm 609.45$ & 1287.14 & 263.93 & 1043.05 & 1531.23 & 12.90 & $.00 *$ \\
\hline
\end{tabular}

Significance level $p \leq 0.05, \quad \quad$ *significant, $95 \%$

C.I. $=95 \%$ Confidence Interval of the Difference

\section{DISCUSSION}

The periodontal pocket harbors subgingivally large number of periodontal pathogens that sometimes resist conventional periodontal treatment and ordinary antimicrobials (Nakao et al 2011). Although mechanical plaque control still is the gold standard in treating periodontal diseases, some limitations exists. The difficult accessibility to deep pockets and the presence of smear layer after instrumentation represents some of these limitations (Lopes et al 2008). Also the bacterial resistance to antibiotics that may be related to the constant exposure to chemicals (Woodford \& Ellington 2007) necessitates the need to find a different treatment modality to enhance the results of periodontal treatment.

Nowadays, different treatment modalities and materials have been introduced in dentistry for periodontal treatment. An alternative periodontal treatment modality with potent antimicrobial effect is the antimicrobial photodynamic therapy (A-PDT) (Wainwright 1998, Fekrazad et al 2017). Studies proved that A-PDT may enhance the results of periodontal treatment when used as adjunctive modality to periodontal debridement by augmenting bacterial eradication and decreasing the bacterial virulence (Rajesh et al 2011, Carrera et al 2016, Kashef \& Hamblin 2017). The development of bacterial resistance to A-PDT seems unlikely as the A-PDT cause complete damage to the bacterial lipopolysaccharides and DNA not just inhibiting the bacterial growth (Konopka \& Goslinski 2007, Costa et al 2011, Parker 2013, Kashef \& Hamblin 2017). Regarding the field of biomaterials used in periodontics nowadays, herbs and medicinal plants have been re-explored being economical, safe and effective in management of many oral diseases (Davies et al 2010, Palombo 2011, Maldupa et al 2012).

In the present study, curcumin had been selected to be used. Besides being anti-inflammatory, antibacterial and safe natural material (Sharma et al 2004, Jurenka 2009), it has many properties of an ideal photosensitizer for eradication of periodontal pathogens. It has the power to form singlet oxygen in an aprotic environment while being highly biocompatible (Araujo et al 2012). Curcumin can absorb blue light and exert strong antibacterial and anti-inflammatory effects (Gupta et al 2013). It has maximum absorption at wavelength range 300500nm (Baltazar et al 2015, Pourhajibagher et al 2016). The light source used in the present study was the light emitting diode (LED) in the wave length 440-480 nm (visible blue light) routinely present in the dental clinic for curing of dental polymers (composite fillings). This was according to previous 
in-vitro studies that showed that this light can activate the curcumin and result in high bacterial reduction with 5 minutes irradiation time (Paschoal et al 2013, Mahdi et al 2015). The light source was held as close as possible to the surgical site to achieve constant power density (Mahdi et al 2015). To the best of our knowledge, the present study was the first to evaluate the clinical and microbiological effectiveness of A-PDT using curcumin and LED as an adjunctive modality with OFD in the management of periodontitis. The present study hypothesized that A-PDT with curcumin in conjunction with OFD could lead to more improvement in the clinical periodontal parameters in addition to greater decrease in the subgingival microbial load when compared to OFD alone.

Strict patient selection criteria were used in the present study to minimize the variables that may have an effect on the results. All the patients were selected to have periodontitis with at least one true periodontal pocket with probing depth greater than or equal $5 \mathrm{~mm}$ to justify the open flap debridement. According to many studies, the presence of residual pockets more than or equal $5 \mathrm{~mm}$ after conventional phase 1 therapy is a risk factor for relapse of the treatment and further disease progression and thus is a clear indication for surgical periodontal therapy (Lindhe et al 1982,Claffey et al 1990, Claffey \& Egelber 1995, Matuliene et al 2008). The present study included periodontal pockets with signs of horizontal bone loss on radiographic examination and thus regenerative procedures were not required and no need for radiographic follow-up. Intraoral bitewing radiographs were used in this study for the detection of interproximal horizontal bone loss. Previous studies showed that the geometric distortion is minimized in bitewing radiograph, thus it is a good option to detect alveolar bone level changes (Pepelassi et al 2000, Gedik et al 2008, Kim et al 2008). All patients in our study were medically free in order to avoid any systemic disorder or medications that may affect the periodontal wound healing. Smokers and pregnant females were excluded from the present study since previous studies have shown that smokers may show different response to periodontal treatment and different subgingival flora compared to non-smokers (Page \& Kornman 1997, Johnson \& Hill 2004) while pregnancy may alter the inflammatory response and progression of periodontal disease without significant concomitant change in plaque index (Gursoy et al 2008, Carrillo et al 2010). The concept of local drug delivery at the periodontal defect allow the achievement of sufficient concentration of the drug at the treated site compared to systemic administration in addition to decreased side effects, dosages and increased patient compliance ( Soskolne 1997, Jorgensen \& Slots 2000). The curcumin locally-placed in the surgical site can easily access the root and bone surface after mechanical debridement (Andersen et al 2007).

Bacterial evaluation was performed in the present study as the bacteria are considered the most important risk factor of the periodontal disease; and there is a strong correlation between the bacterial markers and progression of the periodontal diseases (Slots \&Chen 1999; Charalampakis et al 2013). The PCR is among the most sensitive and rapid methods for assessment of periodontal pathogens prevalence (Ashimoto et al 1996, Sakamoto et al 1999). Real-Time PCR for quantification of the total bacterial load was the selected method in this study for bacterial evaluation regarding its speed, accuracy, sensitivity, repeatability and no cross-reactivity with non-target organisms. It easily quantifies uncultivable, slowly growing and fastidious strains (Loozen et al. 2011, Clais et al. 2015). Endodontic paper points was used in the present study for gingival crevicular fluid (GCF) collection as it is an easy, rapid, non-technique sensitive and precise method for GCF collection in compare to micropipettes or the washing technique (Majeed et al., 2016). The 3-months followup period to reevaluate the clinical periodontal parameters was selected in our study since previous studies showed that A-PDT when used 
as adjunct treatment to mechanical debridement in periodontitis has a significant effect on $\mathrm{PD}$ and $\mathrm{CAL}$ changes at 3-month follow-up than 6-month followup (Azaripour et al 2018). The 1-month follow-up period for microbial re-sampling was selected in our study as previous studies showed that microbial re-colonization of the pocket after therapy may occur by time (Mombelli 2018). Studies showed that if oral hygiene was not ideal after treatment, microbial community to great extent similar to that present before the treatment may reappear. Resampling the subgingival microbiota revealed the reappearance of large number of motile rods and spirochetes in the pocket 8 weeks after periodontal treatment (Magnusson et al 1984).

Statistical analysis showed no significant difference between groups in all the clinical parameters (PI, BI, PD, CAL) at the base line, a finding that ensured comparable treatment outcomes at the follow up. Intragroup statistical analysis of the PI showed that from baseline to 3 months, in each group of the three treatment modalities, there was no significant difference between baseline and follow-up. The area under test was free of plaque at the beginning and the end of the study. Patients were kept under strict maintenance program and performed proper oral hygiene so plaque accumulation was minimal. This allowed proper evaluation of the treatment modalities excluding any additional plaque-inflammatory reactions that could affect treatment outcomes. Regarding the BI, in group $\mathrm{A} \& \mathrm{~B}$, the median value was $=1$ at baseline and decreased to 0 at follow up and intragroup comparison revealed no significant difference between baseline and follow-up in each of the three groups. The slight reduction in bleeding index in group A and B although non-significant may be related to the effect of thorough debridement and removal of local factors which lead to decrease in inflammatory mediators and in turn decrease in bleeding on probing. This is in accordance with previous studies ( Lindhe et al 1984, Yilmaz et al 2002, Carvalho et al 2015). Moreover in group A, the A-PDT used has been shown to reduce bleeding on probing in previous studies (Sigusch et al 2005, Chondros et al 2009, Teymouri et al 2016). While in group B, curcumin has the ability to reduce edema, inflammation and bleeding (Hosadurga et al 2014).

Intragroup statistical analysis showed significant decrease in the PD and CAL after treatment in the three groups. This was in accordance with previous studies that showed that proper OFD with removal of root surface irritants and subgingival microbiota has a great impact on periodontal clinical parameters irrespective of the adjunctive treatment modality used. Resolution of inflammation and the postsurgical shrinkage of the gingival lead to PD reduction and CAL gain with long junctional epithelium formation (Reynolds et al 2003, Carranza et al 2006, Becerik 2011, Graziani et al 2012). In group A, the A-PDT may have augmented the $\mathrm{PD}$ reduction and CAL gain as many previous studies documented that when A-PDT was added to conventional SRP better improvement in PD and CAL was achieved ( Al-zahrani \& Austah 2011, Birang et al 2015, Alwaeli et al 2015, Malgikar et al 2016). In addition a systematic review and meta-analysis by Azaripour in 2018 concluded that A-PDT when used as adjunct treatment to SRP in chronic periodontitis treatment, it has a significant effect on PD reduction and CAL gain at 3 months follow-up. In group B, curcumin may have enhanced the PD reduction and CAL gain owing to its antibacterial effect, wound healing stimulatory effects (Komerik \& Wilson 2002, Magesh et al 2013, Packiavathy et al 2014) and host modulatory effects reported in previous studies (Cho et al 2007, Bakir et al 2016).

Regarding the comparison of percent change in $\mathrm{PD}$ and CAL by time in the three groups, the greatest PD \& CAL percent decrease value was recorded in group A while the least percent decrease was in group $\mathrm{C}$ and there was a significant difference between each 2 study groups. In group $\mathrm{A}$ the mean percent change in PD is - 74.04 and the mean percent 
change in the CAL is $\quad-73.81$ (attachment gain $73.81 \%$ ) while in group $\mathrm{C}$ the mean percent change in PD is -21.89 and the mean percent change in the CAL is -10.00 (attachment gain $10 \%$ ). To the best of our knowledge, no previous studies evaluated A-PDT with curcumin in conjunction with OFD. The greater PD reduction and CAL gain in group A may be related to the production of singlet oxygen and other highly reactive products from curcumin on light activation exerting strong antiinflammatory and antibacterial effects (Dougherty et al 1998, Sharman et al 1999, Cheng et al 2001, Gupta et al 2013). Comparing group B to group $\mathrm{C}$, in group $\mathrm{B}$ the mean percent change in $\mathrm{PD}$ is -45.04 and the mean percent change in the CAL -39.88 ( attachment gain 39.88\%) while in group C the mean percent change in PD is -21.89 and the mean percent change in the CAL is -10.00 ( attachment gain 10\%). The greater PD reduction and CAL gain in group B was in accordance with previous studies which tested curcumin as an adjunct to SRP. Suhag et al in 2007 reported that subgingival irrigation with $1 \%$ curcumin solution lead to greater reduction in bleeding on probing and PD compared to chlorhexidine. Many other studies reported the beneficial role of curcumin in enhancing the periodontal clinical parameters when used as an adjunct to periodontal treatment as mouth wash or subgingival irrigation (Muglikar et al 2013, Gottumukkala et al 2013, Bhatia et al 2014, Anitha et al 2015). This could be explained by curcumin ability to stimulate cellular proliferation and wound healing beside inhibition of periodontal pathogens and pro-inflammatory cytokines (Sidhu et al 1998, Singh et al 2002, Kim et al 2012). Comparing group A to group $\mathrm{B}$, the greater $\mathrm{PD}$ reduction and $\mathrm{CAL}$ gain in group A could be attributed to the A-PDT used in conjunction with the curcumin in group $\mathrm{A}$ while group B involves the use of curcumin without photoactivation. Kolenbrander 2000 reported that many photosensitizers have inherent antimicrobial potential but their bactericidal effect is potentiated by light activation. Although curcumin alone without photoactivation can exert many biological actions but several studies showed that it is highly antibacterial when light activated (Deodhar et al 1980, Cheng et al 2001). The results of our study was in accordance with a study by Sreedhar et al in 2015 which compared the efficiency of curcumin gel with versus without photoactivation as an adjunct to SRP and concluded that photoactivation of curcumin with light $470 \mathrm{~nm}$ raise the beneficial effect of curcumin and the effect is further enhanced when PDT was multiply applied.

Regarding the bacterial evaluation, intragroup statistical analysis revealed that significant reduction in the total bacterial load (TBL) occur in each group after treatment. This is basically related to the OFD surgery which leads to significant bacterial reduction in the periodontal pockets according to many studies (Levy et al 2002, Kyriazis et al 2013, Checchi \& Pascolo 2018). Regarding the mean percent change in the TBL, significant difference was found between each two groups in the study. Comparing group A to B, greater reduction was noticed in group $\mathrm{A}$, this could be related to the additional effect of light activation on the curcumin in group A and this was in accordance with Sreedhar et al 2015. Comparing group $B$ to $C$, greater reduction was noticed in group B which can be explained by previous studies that reported that curcumin have inhibitory effect on growth of periodontal pathogens (Bhatia et al 2014, Izui et al 2016).

Comparing group A to group $\mathrm{C}$, greater reduction was noticed in group A. This may be related to A-PDT used in group A and this is in accordance with the following studies that reported the antibacterial effect of A-PDT. A review study by Malik et al 2010 showed that A-PDT lead to reduction of the periodontal pathogen load in chronic periodontitis. Another study by Araujo et al in 2012 reported significant reduction in salivary microorganisms on testing the effect of A-PDT-curcumin on the saliva samples bacterial content. Also Paschoal et al in 2013 showed that LED-activated curcumin when 
applied to cultures of S.mutans lead to significant decrease in bacterial viability. Moreover, Haag et al 2015 tested the antimicrobial effect of A-PDT in vitro and documented significant decrease in periodontal pathogens by A-PDT. An in-vitro study by Mahdi et al 2015 also showed that blue-light photoactivated-curcumin could lead to significant reduction of periodontal pathogens. In 2016, Najafi et al 2016 also reported that A-PDT with curcumin lead to decrease of A.actinomycetemcomitans CFUs/ml by approximately $65 \%$.

\section{CONCLUSIONS}

Within the limitations of our study and the relatively small sample size, the use of curcumin (2\%) locally in conjunction with OFD may lead to increased enhancement in periodontal clinical parameters and increased reduction in TBL than OFD alone. The additional photoactivation (LED $440-480 \mathrm{~nm}$ ) of the curcumin used in conjunction with OFD may lead to greater PD reduction, greater CAL gain and greater TBL reduction when compared to OFD alone or OFD with curcumin without photoactivation.

\section{RECOMMENDATIONS}

Further studies with greater sample size and more extended follow up periods should be carried out in the future to support the results of the present study and to evaluate the effect of the treatment on the long term. Also, different concentrations of curcumin need to evaluated in future studies to determine the optimal concentration with maximum efficacy.

\section{REFERENCES}

- Abrahamse H, Hamblin MR. New photosensitizers for photodynamic therapy, Biochem. J. 2016; 473: 347-364.

- Adriaens PA, Adriaens LM . Effects of nonsurgical periodontal therapy on hard and soft tissues. Periodontol 2000. 2004;36: 121-145

- Alwaeli HP, Al-Khateeb SN, Al-Sadi A. Long-term clinical effect of adjunctive antimicrobial photodynamic therapy in periodontal treatment: a randomized clinical trial. Lasers Med. Sci. 2015; 30 : 801-807.
- Al-Zahrani MS, Austah ON . Photodynamic therapy as an adjunctive to scaling and root planing in treatment of chronic periodontitis in smokers. Saudi Medical Journal. $2011 ; 1: 1183-1188$

- Ammon HP, Anazodo MI, Safayhi H, Dhawan BN, Srimal RC. Curcumin: A potent inhibitor of leukotriene B4 formation in rat peritoneal polymorphonuclear neutrophils (PMNL) Planta Med. 1992; 58:226.

- Andersen R, Loebel N, Hammond D, Wilson M. Treatment of periodontal diseases by photodisinfection compared to scaling and root planing. J Clin Dent. 2007;18:348.

- Anitha V, Rajesh P, Shanmugam M. Comparative evaluation of natural curcumin and synthetic chlorhexidine in the management of chronic periodontitis as a local drug delivery: A clinical an microbiological study. Indian Journal of Dental Research 2015; 26:53-56.

- $\quad$ Araujo NC, Fontana CR, Gerbi MEM, Bagnato VS. Overall-mouth disinfection by photodynamic therapy using curcumin. Photomed Laser Surg. 2012;30 (2):96-101

- Ashimoto A, Chen C, Bakker I., and Slots J. Polymerase chain reaction detection of 8 putative periodontal pathogens in subgingival plaque of gingivitis and advanced periodontitis lesions. Oral Microbiol. Immunol. 1996; 11: 266-273.

- Azaripour A, Dittrich S, Noorden C, Willershausen B. Efficacy of photodynamic therapy as adjunct treatment of chronic periodontitis: a systematic review and meta-analysis. Lasers Med Sci 2018; 33:407-423

- Bakir B, Ay ZY, Buyukbayram HI, Doguc DK, Bayram D, Candan A, Uskun E. Effect of curcumin on systemic T helper 17 cell response; gingival expressions of interleukin-17 and retinoic acid receptor-related orphan receptor $\gamma \mathrm{t}$ and alveolar bone loss in experimental periodontitis. Journal of Periodontology. 2016; 87:e183-e191.

- Baltazar LM, Ray A, Santos DA, Cisalpino PS, Friedman AJ, Nosanchuk JD. Antimicrobial photodynamic therapy: an effective alternative approach to control fungal infections. Front Microbiol. 2015 13;6:202.

- $\quad$ Becerik S, Turkoglu O, Emingil G, Vural C. Antimicrobial effect of adjunctive use of chlorhexidine mouthrinse in untreated gingivitis: A randomized, placebo-controlled study. APMIS. 2011; 119(6):364-72.

- Bender JS, Thang H, Glogauer M. Novel rinse assay for the quantification of oral neutrophils and the monitoring of chronic periodontal disease. J Periodontal Res. 2006; 41: 214-20.

- $\quad$ Bhatia M, Urolagin SS, Pentyala KB. Novel therapeutic approach for the treatment of periodontitis by curcumin. Journal of Clinical and Diagnostic Research. 2014; 8: 65- 69. 
- $\quad$ Bhatti M, MacRobert A, Meghji S, Henderson B, Wilson M. A Study of the update of Toluidine Blue O by porphyromonas gingivalis and the mechanism of lethal photosensitization. Photochem Photobiol. 1998; 68:370-6.

- $\quad$ Birang R, Shahaboui M, Kiani S, Shadmehr E, Naghsh N. Effect of nonsurgical periodontal treatment combined with diode Laser or photodynamic therapy on chronic periodontitis: A randomized controlled split-mouth clinical trial. Journal of lasers in medical sciences. 2015; 6(3):112-119

- Boehm TK, Ciancio SG. Diode laser activated indocyanine green selectively kills bacteria, J. Int. Acad. Periodontol. 2011;13: 58-63.

- Canakci CF, Canakci V. Pain experienced by patients undergoing different periodontal therapies. J Am Dent Assoc. 2007;138: 1563-73..

- Carneiro LG, Nouh H, Salih E. Quantitative gingival crevicular fluid proteome in health and periodontal disease using stable isotope chemistries and mass spectrometry. J. Clin. Periodontol. 2014;41: 733-747.

- Carranza FA, Newman MG, Takei HH, Klokkevold PR. Carranza's clinical periodontology. 10th edition. 2006

- Carranza FA, Takei HH. The flap technique for pocket therapy, in: M.G. Newman, H.H. Takei, P.R. Klokkevold, F.A. Carranza (Eds.), Clinical Periodontology, 10th edition, Saunders, India, 2010; 937-949.

- Carrera ET, Dias HB, Corbi S, Marcantonio RAC, Bernardi ACA, Bagnato VS, Hamblin MR, Rastelli ANS. The application of antimicrobial photodynamic therapy (aPDT) in dentistry: a critical review, Laser Phys.2016; 26 :12.

- Carrillo de-Albornoz A, Figuero E, Herrera D, BasconesMartínezA. Gingival changes during pregnancy: II. Influence of hormonal variations on the subgingival biofilm. J Clin Periodontol 2010; 37(3):230-40.

- Carvalho VF, Andrade P, Rodrigues MF, Hirata MH, Hirata RD, Pannuti CM, De Micheli, Conde MC, Antimicrobial photodynamic effect to treat residual pockets in periodontal patients: a randomized controlled clinical trial, J. Clin. Periodontol. 2015; $42: 440-447$.

- Caton J. Periodontal diagnosis and diagnostic aids. American Academy of Periodontology. Proceedings of the World Workshop in Clinical Periodontics. Chicago: American Academy of Periodontology 1989;1/5-1-22.

- Charalampakis G, Dahlen G, Carl en A, Leonhardt A. Bacterial markers vs. clinical markers to predict progression of chronic periodontitis: a 2-yr prospective observational study. Eur J Oral Sci 2013; 121: 394-402.

- Chattopadhyay I, Biswas K, Bandyopadhyay U, Banerjee RK. Turmeric and curcumin: Biological actions and medicinal applications. Curr Sci. 2004; 87: 44-54.
- Checchi V, Pascolo G. Microbiological Response to Periodontal Therapy: A Retrospective Study . The Open Dentistry Journal, 2018; 12, 837-845

- Chen WR, Singhal AK, Liu H, Nordquist RE. Antitumor immunity induced by laser immunotherapy and its adoptive transfer, Cancer Res. 2001;61: 459-461.

- Chen WR, Liu H, Ritchey JW, Bartels KE, Lucroy MD, Nordquist RE. Effect of different components of laser immunotherapy in treatment of metastatic tumors in rats, Cancer Res. 2002;62: 4295-4299

- Cheng AL, Hsu CH, Lin JK, Hsu MM, Ho YF, Shen TS, Ko JY, Lin JT, Lin BR, Ming-Shiang W, Yu HS,

- Jee SH, Chen GS, Chen TM, Chen CA, Lai MK, Pu YS, Pan MH, Wang YJ, Tsai CC, Hsieh CY. Phase I clinical trial of curcumin, a chemopreventive agent, in patients with high-risk or pre-malignant lesions. Anticancer Res. 2001;21(4B):2895-2900.

- Chignell CF, Bilski P, Reszka KJ, Motton AG, Sik RH, Dahl TA. Spectral and photochemical properties of curcumin. Photochem Photobiol. 1994;59 (3):295-302.

- Cho JW, Lee KS and Kim CW. Curcumin attenuates the expression of IL-1beta, IL-6, and TNF-alpha as well as cyclin E in TNF alpha-treated HaCaT cells; NF kappaB and MAPKs as potential upstream targets. International Journal of Molecular Medicine. 2007; 19:469-474.

- Chondros P, Nikolidakis D, Christodoulides N, Rössler $\mathrm{R}$, Gutknecht N \& Sculean A. Photodynamic therapy as adjunct to non-surgical periodontal treatment in patients on periodontal maintenance: a randomized controlled clinical trial. Lasers Med Sci. 2009; 24:681-688

- Clais S, Boulet G, Van kerckhoven, Lanckacker E, Delputte P, Maes, Cos P. Comparison of Viable Plate Count, Turbidity Measurement and Real-Time PCR for Quantification of Porphyromonas Gingivalis. Lett Appl Microbiol. 2015; $60: 79-84$.

- Claffey N, Egelberg J. Clinical indicators of probing attachment loss following initial periodontal treatment in advanced periodontit is patients. Journal of clinical periodontology 1995; 22(9):690-6.

- Claffey N, Nylund K, Kiger R, Garrett S, Egelberg J. Diagnostic predictability of scores of plaque, bleeding, suppuration and probing depth for probing attachment loss: $31 / 2$ years of observation following initial periodontal therapy. Journal of clinical per iodontology. 1990; 17(2):108-14.

- Cohen AN, Veena MS, Srivatsan ES and Wang MB. Suppression of interleukin 6 and 8 production in head and neck cancer cells with curcumin via inhibition of Ikappa beta kinase. Archives of Otolaryngology - Head and Neck Surgery 2009; 135:190-197. 
- Costa L, Tomé JP, Neves MG, Tomé AC, Cavaleiro JA, Faustino MA, Cunha, Gomes NC, Almeida A, Evaluation of resistance development and viability recovery by a nonenveloped virus after repeated cycles of Apdt. Antiviral Res.2011; 91 : 278-282.

- Darveau RP, Tanner A, Page RC. The microbial challenge in periodontitis. Periodontol 2000.1997; 14: 12-32

- Davies R, Scully C, Preston AJ. Dentifrices--an update. Med Oral Patol Oral Cir Bucal, 2010; 15: e976-982.

- Deniell MD, Hill JS. A history of Photodynamic therapy. Aust N Z J Surg. 1991; 61:340-8. .

- Deodhar SD, Sethi R, Srimal RC. Preliminary study on antirheumatic activity of curcumin (diferuloyl methane). Indian J Med Res. 1980; 71:632-634.

- Dougherty TJ, Gomer CJ, Henderson BW, Jori G, Kessel D, Korbelik M, Moan J, Peng Q . Review. Photodynamic therapy. J Natl Cancer Inst. 1998; 90:889-905

- Fahey AJ, Adrian Robins R, Constantinescu CS. Curcumin modulation of IFN-beta and IL-12 signal ling and cytokine induction in human T cells. Journal of Cellular and Molecular Medicine 2007; 11:1129-1137.

- Fekrazad R, Khoei F, Bahador A, Hakimiha N. Photo-activated elimination of Aggregatibacter actinomycetemcomitans in planktonic culture: Comparison of photodynamic therapy versus photothermal therapy method. Photodiagnosis Photodyn Ther. 2017;19:28-32.

- Feuerstein O, Persman N, Weiss EI. Phototoxic effect of visible light on Porphyromonas gingivalis and Fusobacterium nucleatum: an in vitro study. Photochem Photobiol 2004; 80(3):412-415.

- $\quad$ Flemmig TF, Beikler T. Control of oral biofilms. Periodontology 2000. 2011; 55(1):9-15.

- Gedik R, Marakoglu I, Demirer S. Assessment of alveolar bone levels from bitewing, periapical and panoramic radiographs in periodontitis patients. West Indian Med J 2008;57:410-3

- Glavind L, Loe H. Errors in the clinical assessment of periodontal destruction. J Periodontol Res.1967;2: 180- 184.

- Glick M, Greenberg MS, Ship JA: Introduction to Oral Medicine and Oral Diagnosis: Evaluation of the dental patient. In Burket s oral medicine. eleventh ed, 2008

- Gottumukkala SN, Koneru S, Mannem S, Mandalapu N. Effectiveness of sub gingival irrigation of an indigenous $1 \%$ curcumin solution on clinical and microbiological parameters in chronic periodontitis patients: A pilot randomized clinical trial. Contemp Clin Dent. 2013;4:186-91.

- Grant WE, Hopper C, Speight PM, Bown SG. Photodynamic therapy, an effective, but non-selective treatment for superficial cancers of the oral cavity. Int J Cancer. 1997;71:937-42
- Graziani F, Gennai S, Cei S, et al. Clinical performance of access flap surgery in the treatment of the intrabony defect. A systematic review and metaanalysis of randomized clinical trials. J Clin Periodontol. 2012; 39:145-156.

- Greenstein G .Nonsurgical periodontal therapy in 2000:a Literature review. J Am Dent Assoc. 2000; 131:1580-1592

- Gulcubuk A, Altunatmaz K, Sonmez K, Haktanir-Yatkin D, Uzun H, Gurel A. Effects of curcumin on tumour necrosis factor-alpha and interleukin-6 in the late phase of experimental acute pancreatitis. J Vet Med A Physiol Pathol Clin Med. 2006; 53:49-54.

- Gupta SC, Patchva S, Aggarwal BB. Therapeutic roles of curcumin: lessons learned from clinical trials. AAPS J 2013;15(1):195-218.

- Gursoy M, Pajukanta R, Sorsa T, KönönenE. Clinical changes in periodontium during pregnancy and post-partum. J Clin Periodontol 2008; 35(7):576-83.

- Haag PA, Steiger-Ronay V, Schmidlin PR. The invitro antimicrobial efficacy of PDT against periodontopathogenic bacteria. Int J Mol Sci. 2015; 16(11):27327-38.

- Haffajee, Anne D, Sigmund S Socransky, and John C Gunsolley. Systemic Anti-Infective Periodontal Therapy. A Systematic Review Systemic Anti-Infective Therapy. Annual Periodontology 2003; 8 (1): 115-81.

- Haffajee AD. Systemic antibiotics: To use or not to use in the treatment of periodontal infections: That is the question. J Clin Periodontol. 2006; 33: 359-61.

- Hamblin MR, Hasan T. Photodynamic therapy: A new antimicrobial approach to infectious diseases. Photochem Photobiol Sci. 2004; 3: 436-50.

- Hatcher H, Planalp R, Cho J, Torti FM, Torti SV. Curcumin: from ancient medicine to current clinical trials. Cell Mol Life Sci. 2008;65(11):1631-1652.

- Heitz-Mayfield LJA, Lang NP. Surgical and nonsurgical periodontal therapy. Learned and unlearned concepts. Periodontol2000. 2013:62:218-231

- Hong J, Bose M, Ju J, et al. Modulation of arachidonic acid metabolism by curcumin and related beta diketone derivatives: Effects on cytosolic phospholipase A(2), cyclooxygenases and 5-lipoxygenase. Carcinogenesis 2004; 25:1671-1679.

- Hosadurga RR, Rao SN, Jose J, Rompicharla NC , Shakil M, and Shashidhara. Evaluation of the efficacy of $2 \%$ curcumin gel in the treatment of experimental periodontitis. . Pharmacognosy Res. 2014; 6(4): 326-333

- How KY, Song KP, Chan KG . Porphyromonas gingivalis: an overview of periodontopathic pathogen below the gum line. Front Microbiol. 2016; 7:53 
- Hu P, Huang P and Chen MW. Curcumin reduces Streptococcus mutans biofilm formation by inhibiting sortase A activity. Archives of Oral Biology 2013a; 58:343-348

- $\quad$ Izui S, Sekine S, Maeda K, et al. Antibacterial activity of curcumin against periodontopathic bacteria. Journal of Periodontology 2016; 87:83-90.

- Johnson GK, Hill M. Cigarette smoking and the periodontal patient. J Periodontol 2004; 75:196-209.

- Jorgensen MG, Slots J. Responsible use of antimicrobials in periodontics. J Calif Dent Assoc. 2000; 28:185-93

- Jurenka JS. Anti-inflammatory properties of curcumin, a major constituent of Curcuma longa: A review of preclinical and clinical research. Altern Med Rev. 2009;14:141-53.

- Juzeniene A, Juzenas P, Ma LW, Iani V, Moan J. Effectiveness of different light Sources for 5 - aminolevulinic acid photodynamic therapy. Lasers Med Sci. 2004;19:139-49.

- Kaldahl WB, Kalkwarf KL, Patil KD, Dyer JK, Bates RE. Evaluation of four modalities of periodontal therapy. J Periodontol. 1988;59:783-93.

- Kashef N, Hamblin MR.Can microbial cells develop resistance to oxidative stress in antimicrobial photodynamic inactivation? Drug Resist. Updat. 31. 2017; 31-42.

- $\quad$ Khopde SM, Priyadarsini KI, Palit DK, Mukherjee T. Effect of solvent on the excited-state photophysical properties of curcumin. Photochem Photobiol. 2000;72(5):625-631.

- Kim GY, Kim KH, Lee SH, Yoon MS, Lee HJ, Moon DO. Curcumin inhibits immunostimulatory function of dendritic cells: MAPKs and translocation of NF-kappa B as potential targets. J Immunol. 2005 ;174:8116-24.

- $\quad$ Kim TS, Obst C, Zehaczek S, Geenen C. Detection of bone loss with different $\mathrm{X}$-ray techniques in periodontal patients. J Periodontol 2008;79:1141-9.

- $\quad$ Kim KH, Lee EN, Park JK, Lee JR, Kim JH. Curcumin attenuates TNF- induced expression of intercellular adhesion molecule-1, vascular cell adhes ion molecule- 1 and proinflammatory cytokines in human endometriotic stromal cells. Phytother Res 2012; 26: 1037-1047.

- Kolenbrander PE. Oral microbial communities: biofilms, interactions, and genetic systems. Annu Rev Microbiol. 2000;54:413-437.

- $\quad$ Komerik N, Wilson M, Poole S. The effect of photodynamic action on two virulence factors of gram - negative bacteria. Photochem Photobiol. 2000;72:676-80.

- Komerik N, Wilson M. Factors influencing the susceptibility of Gram-negative bacteria to toluidine blue O-mediated lethal photosensitization. J Appl Microbiol. 2002;92:618-23.

- Komerik N, MacRobert AJ. Photodynamic therapy as an alternative antimicrobial modality for oral infections. J Environ Pathol Toxicol Oncol 2006;25(1-2):487-504.
- $\quad$ Kommerein N, Stumpp SN, Müsken M, Ehlert N, Winkel A, Häussler S. An oral multispecies biofilm model for high content screening applications. PLoS One. 2017;12(3).

- Konopka K, Goslinski T, Photodynamic therapy in dentistry, J. Dent. Res. 2007;86: 694-707.

- Koo H, Allan RN, Howlin RP, Stoodley P, Hall-Stoodley L. Targeting microbial biofilms: current and prospective therapeutic strategies. Nat Rev Microbiol. 2017;15:740-755.

- Kornman KS, Page RC, Tonetti MS. The host response to the microbial challenge in periodontitis: Assembling the players. Periodontol 2000. 1997;14:33-53.

- Kyriazis T, Gkrizioti S, Tsalikis L, Sakellari D, Deligianidis A, Konstantinidis A. Immunological and microbiological findings after the application of two periodontal surgical techniques: A randomized, controlled clinical trial. J Clin Periodontol. 2013; 40(11): 1036-42.

- $\quad$ Lambrecht B, Mohr H, Knuver - Hopf J, Schmitt H. Photoinactivation of viruses in human fresh plasma by phenothiazine dyes in Combination with visible light. Vox Sang. 1991;60:207-13.

- Lampe V, Milobedzka J. Studies on curcumin. Ber Dtsch Chem Ges. 1913;46:2235-7.

- Levy RM, Giannobile WV, Feres M, Haffajee AD, Smith C, Socransky SS. The effect of apically repositioned flap surgery on clinical parameters and the composition of the subgingival microbiota: 12-month data. Int J Periodontics Restorative Dent 2002; 22(3): 209-19.

- $\quad$ Lindhe J, Socransky SS, Nyman S, Haffajee A, Westfelt E. -Critical probing depths-in periodontal therapy. Journal of Clinical Periodontology. 1982 ;9(4):323-36.

- $\quad$ Lindhe J, Westfelt E, Nyman S, Socransky S, Haffajee $\mathrm{AD}$, Long-term effect of surgical/non-surgical treatment of periodontal disease, J. Clin. Periodontol.1984; 11 :448458.

- $\quad$ Loe \& Silness. The Gingival Index, the Plaque Index and the Retention Index Systems. Journal of Periodontology, 1967; 38: 6; 610-616.

- Loozen G, Boon N, Pauwels M, Quirynen M, Teughels W. live / dead real-time polymerase chain reaction to Assess new therapies against dental plaque-related pathologies. Molecular Oral Microbiology.2011; 26 (4): 253-61.

- Lopes B, Marcantonio R, Thompson GMA, Neves LHM, Theodoro L . "Short-Term Clinical and Immunologic Effects of Scaling and Root Planing With Er:YAG Laser in Chronic Periodontitis." Journal of Periodontology 2008; 79 (7): 1158-67.

- Lopez NJ, Gamonal JA, Martinez B. Repeated metronidazole and amoxicillin treatment of periodontitis. A followup study. J Periodontol. 2000;71:79-89. 
- Magesh H, Kumar A, Alam A, Priyam, Sekar U, Sumantran VN, Vaidyanathan R. Identification of natural compounds which inhibit biofilm formation in clinical isolates of Klebsiella pneumoniae. Indian Journal of Experimental Biology 2013; 51:764-772.

- Magnusson I, Lindhe J, Yoneyama T, Liljenberg B. Recolonization of a subgingival microbiota following scaling in deep pockets. J Clin Periodontol 1984: 11: 193-207.

- Mahdi Z, Habiboallh G, Mahbobeh N, Mina Z, Majid Z, Nooshin A . Lethal effect of blue light-activated hydrogen peroxide, curcumin and erythrosine as potential oral photosensitizers on the viability of Porphyromonas gingivalis and Fusobacterium nucleatum. Laser Therapy 2015; 24(2): 103-111

- $\quad$ Majeed ZN, Philip K, Alabsi AM, Pushparajan S, Swaminathan D. Identification of Gingival Crevicular Fluid Sampling, Analytical Methods, and Oral Biomarkers for the Diagnosis and Monitoring of Periodontal Diseases: A Systematic Review. Hindawi Publishing Corporation. Disease Markers Volume 2016; Article ID 1804727:1-23

- Maldupa I, Brinkmane A, Rendeniece I, Mihailova A. Evidence based toothpaste classification, according to certain characteristics of their chemical composition. Stomatologija 2012; 14: 12-22.

- Malgikar S, Reddy SH, Sagar SV, Satyanarayana D, Reddy GV, JosephinJJ, Clinical effects of photodynamic and low-level laser therapies as an adjunct to scaling and root planing of chronic periodontitis: a split-mouth randomized controlled clinical trial, Indian J. Dent. Res. 2016;27: 121-126.

- Malik R, Manocha A, Suresh DK. Photodynamic therapy - A strategic review. Indian J Dent Res. 2010; 21:285-91.

- Matuliene G, Pjetursson BE, Salvi GE, Schmidlin K, Bragger U, Zwahlen M, Lang NP. Influence of residual pockets on progression of periodontitis and tooth loss: results after 11 years of maintenance. Journal of clinical periodontology 2008; 35(8):685-95.

- Meyer-Betz F. Study of the biological (photodynamic) effect of hematoporphyrin and others Derivatives of the blood and bile pigments. Dtsch Arch Klin Med 1913; 112:476-503

- Millson CE, Wilson M, Macrobert AJ, Bedwell J, Brown SG. The killing of helicobacter pylori by low-power laser light in the presence of a photosensitizer. J Med Microbiol. 1996;44:245-52.

- Mombelli A. Microbial colonization of the periodontal pocket and its significance for periodontal therapy Periodontology 2000. 2018;76: 85-96

- Moon DO, Kim MO, Choi YH, Park YM, Kim GY. Curcumin attenuates inflammatory response in IL-1beta-in- duced human synovial fibroblasts and collagen-induced arthritis in mouse model. Int Immunopharmacol. 2010; 10:605-10.

- Muglikar S, Patil KC, Shivswami S, Hegde R. Efficacy of curcumin in the treatment of chronic gingivitis: A pilot study. Oral Health Prev Dent. 2013;11:81-6

- Mun SH, Kim HS, Kim JW, Ko NY, Kim do K, Lee BY. Oral administration of curcumin suppresses production of matrix metalloproteinase (MMP)-1 and MMP-3 to ameliorate collagen-induced arthritis: Inhibition of the PKCdelta/ JNK/c-Jun pathway. J Pharmacol Sci. 2009;111:13-21.

- Najafi S, Khayamzadeh M, Paknejad M, Poursepanj G, Kharazi Fard MJ, Bahador A. An in vitro comparison of antimicrobial effects of curcumin-based photodynamic therapy and chlorhexidine, on Aggregatibacter actinomycetemcomitans. J Lasers Med Sci. 2016;7(1):21-5.

- Nakao R, Takigawa S, Sugano N, Koshi R, Ito K, Watanabe $\mathrm{H}$, et al. "Impact [21] of minocycline ointment for periodontal treatment of oral bacteria." J Infect Dis. 2011; 64(2):156-60

- $\quad$ Negi PS, Jayaprakasha GK, Jagan Mohan Rao L and Sakariah, KK. Antibacterial activity of turmeric oil: A by product from curcumin manufacture. The Journal of Agricultural and Food Chemistry 1999; 47:4297-4300

- Nesi-Reis V, Lera-Nonose DSSL, Oyama J, et al., Contribution of photodynamic therapy in wound healing: a systematic review, Photodiagnosis Photodyn. Ther. 2018; 21: 294-305.

- Novak MJ, Novak KF, Smoking in periodontal disease. in: M.G. Newman, H.H. Takei, P.R. Klokkevold, F.A. Carranza (Eds.), Clinical Periodontology, 10th edition, Saunders, India, 2010: 251-258.

- $\quad$ O’Neill JF, Hope CK, Wilson M. Oral bacteria in multispecies biofilms can be killed by red light in the presence of toluidine blue. Lasers Surg Med 2002;31(2):86-90

- Packiavathy IA, Priya S, Pandian SK and Ravi AV. Inhibition of biofilm development of uropathogen by curcumin - An anti-quorum sensing agent from Curcuma longa. Food Chemistry 2014; 148:453-460.

- $\quad$ Page R C \& Kornman K S. The pathogenesis of human periodontitis: an introduction. Periodontology 2000; 1997, 14, 9- 11 .

- $\quad$ Page RC, Offenbacher S, Schroeder HE, Seymour GJ, Kornman KS . Advances in the pathogenesis of periodontitis: summary of developments, clinical implications and future directions. Periodontol 2000. 1997; 14:216-248

- Palombo EA. Traditional medicinal plant extracts \& natural products with activity against oral bacteria: potential application in the prevention \& treatment of oral diseases. Evid Based Complement Alternat Med 2011; 6:803-854. 
- $\quad$ Parker S, The use of diffuse laser photonic energy and indocyanine green photosensitizer as an adjunct to periodontal therapy, Br. Dent. J. 2013;215:167-171.

- Paschoal MA, Tonon CC, Spolidório DM, BagnatoVS, Giusti JS,Santos-Pinto L. Photodynamic potential of curcumin and blue LED against Streptococcus mutans in a planktonic culture. Photodiagnosis Photodyn Ther 2013;10(3):313-319.

- Pepelassi EA, Tsiklakis K, Diamanti-Kipioti A. Radiographic detection and assessment of the periodontal endosseous defects. J Clin Periodontol 2000;27:224-30.

- Pourhajibagher M, Chiniforush N, Parker S, Shahabi S, Ghorbanzadeh R, Kharazifard MJ. Evaluation of antimicrobial photodynamic therapy with indocyanine green and curcumin on human gingival fibroblast cells: An in vitro photocytotoxicity investigation. Photodiagnosis Photodyn Ther. 2016;15:13-8.

- Rajesh S, Koshi E, Philip K, Mohan A, Antimicrobial photodynamic therapy: an overview, J. Indian Soc. Periodontol. 2011;15: 323-327.

- Ranjan D, Chen C, Johnston TD, Jeon H and Nagab hushan M. Curcumin inhibits mitogen stimulated lymphocyte proliferation, NF-kappaB activation, and IL-2 signaling. Journal of Surgical Research 2004;121:171-177.

- Reynolds MA, Aichelmann-Reidy ME, Branch-Mays GL, Gunsolley JC. The efficacy of bone replacement grafts in the treatment of periodontal osseous defects. A systematic review. Ann Periodontol 2003;8:227-265.

- $\quad$ Rivera MF, Lee JY, Aneja M, Goswami V, Liu L, Velsko IM. Polymicrobial infection with major periodontal pathogens induced periodontal disease and aortic atherosclerosis in hyperlipidemic ApoE(null) mice. PLoS One. 2013;8:2

- $\quad$ Sakamoto, M., Takeuchi, Y., Umeda, M., Ishikawa, I., Benno, Y, and Nakase, T.. Detection of Treponema socranskii associated with human periodontitis by PCR. Microbiol. Immunol.1999; 43: 485-490.

- Salva KA. Photodynamic therapy: Unapproved uses, dosages or indications. Clin Dermatol. 2002;20:571-81.

- Schenk G, Flemming TF, Lob S, Ruckdeschel G, Hickel R. Lack of antimicrobial effect on periodontopathic bacteria by ultrasonic and sonic scalers in vitro, J. Clin. Periodontol. .2000; 27:116-119.

- Sharma RA, Euden SA, Platton SL, Cooke DN, Shafayat A, Hewitt HR, Marczylo TH, Morgan B, Hemingway D, Plummer SM, Pirmohamed M, Gescher AJ, Steward WP. Phase I clinical trial of oral curcumin: Biomarkers of systemic activity and compliance. Clinical Cancer Research 2004; 15:6847-6854.

- Sharma S, Shwetha P, Upendra KJ. Development and evaluation of topical Curcumin from different combina- tion of polymers formulation and evaluation of herbal gel. Int J Pharm Pharm Sci. 2012;4:4452-6.

- Sharman WM, Allen CM, van Lier JE Photodynamic therapeutics: basic principles and clinical applications. Drug Discov 1999; 4:507-517

- Sharwani A, Jerjes W, Salih V, MacRobert AJ. Fluorescence Spectroscopy combined with 5 aminolevulinic acid - induced protoporphyrin IX fluorescence in detecting oral premalignancy. J Photochem Photobiol B. 2006;83:27-33.

- $\quad$ Sidhu GS, Singh AK, Thaloor D, Banaudha KK, Patnaik GK, Srimal RC, Maheshwari RK Enhancement of wound healing by curcumin in animals. Wound Repair Regen. 1998; 6(2):167-77.

- $\quad$ Sigusch BW, Pfitzner A, Albrecht V, Glockmann E Efficacy of photodynamic therapy on inflammatory signs and two selected periodontopathogenic species in a beagle dog model. J Periodontol. 2005; 76:1100-1105

- Silness J, Löe H. Correlation between oral hygiene \& periodontal condition. Acta Odontol Scand 1964; 22: 121-135.

- $\quad$ Singh R, Chandra R, Bose M, Luthra PM. Antibacterial activity of curcumin longa rhizome extract on penopathogenic bacteria. Curr Sci. 2002; 83:737-40.

- Slots J, Chen C. The oral microflora and human periodontal diseases. Medical importance of the normal microflora. Springer. 1999

- $\quad$ Soskolne WA. Subgingival delivery of therapeutic agents in the treatment of periodontal diseases. Critical Re-views in Oral Biology and Medicine 1997; 8:164-174.

- Soukos NS, Som S, Abernethy AD, Ruggiero K, dunham J, Lee C, Doukas AG, Goodson JM. Phototargeting oral black-pigmented bacteria. Antimicrob agents Chemother 2005;49:1391-1396.

- Sreedhar A, Sarkar I, Barmappa R Comparative evaluation of the efficacy of curcumin gel with and without photo activation as an adjunct to scaling and root planing in the treatment of chronic periodontitis: A split mouth clinical and microbiological study, J Nat Sci Bio Med 2015; s 102-s109

- Stone, Patricia W. Popping the (PICO) Question in Research and Evidence-Based Practice. Applied Nursing Research. 2002; 16 (2): 197-98

- Suhag A, Dixit J, Dhan P. Role of curcumin as a sub gingival irrigant : A pilot study. J Perio 2007;4(2):115- 21.

- $\quad$ Syed SA, Loesche WJ. "Survival of Human Dental Plaque Flora in Various Transport Media." Applied Microbiology. 1972; 24 (4): 638-44.

- Takasaki AA, Aoki A, Mizutani K, Schwarz F, Sculean A, Wang CY. Application of antimicrobial photodynamic therapy in periodontal and peri-implant diseases. Periodontol 2000. 2009;51:109-40. 
- Teymouri F, Farhad S, Golestaneh H, The effect of photodynamic therapy and diode laser as adjunctive periodontal therapy on the inflammatory mediators levels in gingival crevicular fluid and clinical periodontal status, J. Dent. 2016; 17 : 226-232.

- Theodoro LH, Silva SP, Pires JR, Soares GHG, Pontes AF, Zuza P , Correa de Toledo BE, Garcia VG. Clinical and microbiological effects of photodynamic therapy associated with nonsurgical periodontal treatment. A 6-month follow-up. Lasers Med Sci. 2012;27(4):687-93

- Tyagi P, Singh M, Kumari H, Kumari A and Mukhopadhyay K. Bactericidal activity of curcumin I is associated with damaging of bacterial membran PLoS ONE 2015; 10(3):e121-313

- Vera-Ramirez L, Pérez-Lopez P, Varela-Lopez A, RamirezTortosa M, Battino M, Quiles JL. Curcumin and liver disease. Biofactors. 2013;39(1):88-100.
- Wainwright M. Photodynamic antimicrobial chemotherapy (PACT) J Antimicrob Chemother.1998; 42:13-28.

- Wassall, Rebecca R., and Philip M. Preshaw.. "Clinical and Technical Considerations in the Analysis of Gingival Crevicular Fluid.” Periodontology 2000. 2016; 70 (1): 65-79.

- Woodford N, Ellington M, The emergence of antibiotic resistance by mutation. Clin Microbiol Infect.2007;13: $5-18$.

- Wright LE, Frye JB, Gorti B, Timmermann BN, Funk JL. Bioactivity of turmeric-derived curcuminoids and related metabolites in breast cancer. Curr Pharm Des. 2013;19(34):6218-25.

- Yilmaz S, Kuru B, Kuru L, Noyan U, Argun D, Kadir $\mathrm{T}$, Effect of gallium arsenide Diode laser on human periodontal disease: a microbiological and clinical study, Lasers Surg. Med.2002; $30: 60-66$. 